\title{
Thermodynamically Constrained Averaging Theory Approach for Modeling Flow and Transport Phenomena in Porous Medium Systems: 8. Interface and Common Curve Dynamics
}

\author{
William G. Gray ${ }^{*}$ and Cass T. Miller \\ Department of Environmental Sciences and Engineering, University of North Carolina, Chapel \\ Hill, North Carolina 27599-7431, USA
}

\begin{abstract}
This work is the eighth in a series that develops the fundamental aspects of the thermodynamically constrained averaging theory (TCAT) that allows for a systematic increase in the scale at which multiphase transport phenomena is modeled in porous medium systems. In these systems, the explicit locations of interfaces between phases and common curves, where three or more interfaces meet, are not considered at scales above the microscale. Rather, the densities of these quantities arise as areas per volume or length per volume. Modeling of the dynamics of these measures is an important challenge for robust models of flow and transport phenomena in porous medium systems, as the extent of these regions can have important implications for mass, momentum, and energy transport between and among phases, and formulation of a capillary pressure relation with minimal hysteresis. These densities do not exist at the microscale, where the interfaces and common curves correspond to particular locations. Therefore, it is necessary for a well-developed macroscale theory to provide evolution equations that describe the dynamics of interface and common curve densities. Here we point out the challenges and pitfalls in producing such evolution equations, develop a set of such equations based on averaging theorems, and identify the terms that require particular attention in experimental and computational efforts to parameterize the equations. We use the evolution equations developed to specify a closed two-fluid-phase flow model.
\end{abstract}

\section{Keywords}

TCAT; Evolution equations; Interfacial area; Interfacial dynamics; Dynamic capillary pressure

\section{Introduction}

The thermodynamically constrained averaging theory (TCAT) has been introduced in a series of manuscripts as a set of mathematical tools for rigorously increasing the scale at which a problem of interest is considered. Emphasis has been on porous medium systems. The method begins with a set of equations formulated at the microscale (i.e., a continuum scale at which all phase boundaries are explicitly defined); and then makes use of averaging

\footnotetext{
(C) 2010 Elsevier Ltd. All rights reserved.

*Corresponding author, GrayWG@unc.edu (William G. Gray,), casey_miller@unc.edu.

Publisher's Disclaimer: This is a PDF file of an unedited manuscript that has been accepted for publication. As a service to our customers we are providing this early version of the manuscript. The manuscript will undergo copyediting, typesetting, and review of the resulting proof before it is published in its final citable form. Please note that during the production process errors may be discovered which could affect the content, and all legal disclaimers that apply to the journal pertain.
} 
and integration theorems to increase the scale to one suitable for analysis of the problem at a larger scale of interest. Most of the attention has been focused on the macroscale in which the porous medium system may be considered as composed of overlapping continua, but integration to the megascale, or system scale, has also been considered [28]. The equations that are averaged are mass, momentum, and energy conservation laws, entropy balances, and thermodynamic descriptions appropriate for the volumes of the phases, the interfaces between phases, the common curves where three phases meet, and common points where four or more phases may come together. These different region types are collectively referred to as entities. The equations for the interface and common curve entities are not just the jump conditions that are often applied at these locations, but are full dynamic conservation equations. Because integration is performed to increase the scale at which the problem is considered, well-defined relations between microscale and larger-scale variables are obtained, which make the resultant equations amenable to analysis and parameterization based on small-scale physical and computational experiments.

Previous papers in the series have provided the background and overview of the method [25], available and additional averaging theorems employed in TCAT [53], application to flow [26] and transport [27,54] in single-fluid-phase systems, development of flow equations for two-fluid-phase flow [39], and derivation of system-scale, or megascale, equations for single-fluid-phase systems [28]. It is widely recognized that the distribution of the phases in these porous medium systems is of significance in describing the system properties and behavior. In many cases, the solid phase can be considered to be relatively immobile, which simplifies the problem in that the solid deformation and movement is accounted for by storage coefficients and the effective stress. When two or more fluid phases are present, the movement of the interfaces between the fluids and the spreading of fluids over the solid surface are dynamic features of the system that impact behavior and complicate the modeling process.

In the century and a half in which efforts have been made to model porous medium flow problems, the issue of redistribution of fluid phases has been largely confined to determination of the fluid saturations, the fraction of pore space occupied by each fluid. In part this situation is due to the fact that fluid saturations can be measured relatively easily, while more esoteric quantities such as interfacial areas between phases per volume, common curve length per volume, and fraction of the solid surface in contact with a particular fluid phase are more difficult to observe than fluid saturations. The experimental deficits have led to both expanding efforts to obtain information about the details of fluid and solid distributions within porous medium systems and the inclination to formulate theories that do not rely on this information. The former approach has provided possibilities for improved physical understanding and improved models; the latter approach has encouraged stagnation of thought and acceptance of the inadequacies of current understanding.

Recently, photon attenuation and nuclear magnetic resonance imaging methods have emerged as primary tools to observe microscale systems [12,13,18,32,40,44,76,83]. In particular, leading porous medium experimental groups are routinely using high-flux photon sources from a synchrotron beam line to rapidly image porous medium systems with high resolution. These methods have been used to image not only pore structure but also twophase flow features such as fluid distributions, entrapped fluids, curvatures of interfaces, and contact angles [1,2,86-89].

Microscale pore-network models have been developed to model many different processes, including single-phase flow and transport [9,79]; aspects of two-fluid-phase flow such as capillary pressure-saturation-interfacial area relations $[20,61,68]$, fluid entrapment $[35,36,46,47]$, dissolution [16,90], and dispersion [7,75]; and some aspects of three-fluid 
phase flow $[8,34,65,72]$. Similarly, the lattice-Boltzmann method (LBM) has emerged as an attractive microscale simulation method for many types of fluid flows and has become an indispensable tool for the study of porous medium physics $[45,62,63,77]$. For multiphase systems, the LBM avoids difficulties associated with the continuum description of interfaces by introducing a quasi-molecular description of the interfacial physics [33,71]. The LBM is established as a reliable way to obtain permeability values in synthesized or imaged porous medium systems; and simulations of complex multiphase displacement have been shown to agree with detailed, highly resolved experiments $[10,70,78,84]$. The efficacy of the LBM has led to broad efforts to improve computational algorithms [5,14,50,51,73,81,82], accuracy of boundary conditions $[15,19,64]$, and thermodynamic consistency [42,67], in addition to producing a variety of schemes to model processes ranging from single-phase flow to reactive mixtures and multiphase systems $[17,66,69,80]$.

A significant problem with describing multiphase flow and transport in porous medium systems is that specification of fluid saturations does not provide a sufficient amount of information about the morphological and topological state of the system to yield high fidelity, first principles models. At the microscale, one can observe boundaries between fluids, fractions of the solid surface wetted by each fluid, curvature of fluid-fluid interfaces that is proportional to the capillary pressure, and the existence and curvature of common curves. When systems are studied at a larger scale, as is necessary if one is to model fieldscale behavior, this detail is lost. The best one can hope to do is describe these higher order measures of the morphology and topology of the phase distributions in some average sense. Thus, instead of describing the precise locations of phases at the microscale, one makes use of the volume fraction at the macroscale. This quantity only describes the fraction of a volume occupied by a phase. It does not differentiate between elements of a fluid that are disconnected from each other as opposed to a phase that is fully connected. If we do not consider characteristic measures of interfacial areas and common curves in a macroscale formulation, we are restricted in our ability to describe system properties. For example, capillary pressure at the microscale for a two-fluid-phase porous medium system is equal to the surface tension of the fluid-fluid interface multiplied by the interfacial curvature of that interface. Thus to define capillary pressure at the macroscale, we need some surrogate that accounts for average curvature. Such a quantity is typically not available, so the approach has been to settle for expressions of capillary pressure as a function of saturation, the available parameter. As is well known, success of this approach is mixed with one major problem being that capillary pressure is not a single-valued function of saturation but is rather an infinite valued hysteretic relation dependent upon the saturation history of the system. In effect with this common approach, the saturation history is being used as a surrogate for other measures of the morphology and topology of the fluid distributions.

The search for additional information that can eliminate, or at least reduce, hysteresis should begin with macroscale quantities that arise naturally when microscale equations are transformed to macroscale forms. First among these is the interfacial area per volume. A simple thought experiment confirms this notion. Consider two porous medium systems with identical solid phases and with two identical fluid phases present. Let the saturations in the two systems be the same. However, let one fluid in the first system be more dispersed such that the system has more interfacial area between fluids than the other. Since the saturations in the two systems are the same, curvature of the interfaces in the more dispersed system will be higher. Thus, although the saturations are the same, the capillary pressure in the two systems will be different. Thus inclusion of interfacial area per volume as an additional macroscale independent variable on which capillary pressure depends should be helpful in building a surrogate macroscale variable to account for interfacial curvature. Note that this extension makes use of interfacial area per volume as a separate independent variable, not as a variable that itself depends on saturation. Further specification of multiphase system 
behavior can be achieved if one has information on the representation of the spreading of a wetting fluid over a solid surface. Macroscale variables that describe this are the fraction of the solid surface in contact with each fluid phase and the common curve length per volume.

As overhead to the process of including interfacial area density, wetted fraction of the solid phase, and common curve density in the modeling process, there is a need for evolution equations for these quantities. These equations must be based on changes in the morphology and topology of the phases. They are not conservation equations in that, for example, area per volume is not a conserved quantity although the mass, momentum, and energy associate with that area are conserved quantities with corresponding surface-based conservation equations. Thus expressions must be developed that account for the differential geometry of the additional variables. These are, of necessity, approximate expressions that do not carry the cachet of an immutable conservation principle. As a result of being approximations, such evolution equations are subject to improvement as fundamental insight evolves. Modern experimental imaging methods and high-resolution microscale simulation approaches are tools that can hasten such advances.

The overall goal of this work is to advance the modeling of interfaces and common curves in two-fluid-phase porous medium systems. The specific objectives of this work are: (1) to review existing approaches for modeling areas, (2) to develop an improved set of evolution equations for specific interfacial areas and common curve lengths based upon averaging theorems and a clearly specified set of approximations, (3) to evaluate a recently proposed model for evolving interfacial areas in light of the model developed in this work, (4) to use the developed area model to close a two-fluid-phase flow model, and (5) to discuss remaining open issues and potential paths for resolving these issue.

\section{Background}

For analysis of fluid flow in porous medium systems, equations for the evolution of interfacial area and common curve densities are needed at the macroscale. For consistency with the TCAT approach, these equations should be obtained by averaging from the microscale to the macroscale through the use of averaging theorems. Thus, it is useful to examine approaches for obtaining such evolution equations that have appeared in the literature and which serve to place the present work in context. Before proceeding, we note the warning that in spite of numerous attempts, no general strategy has emerged for solving the equations of fluid mechanics to obtain the shapes of fluid interfaces [52]. That statement was made in regard to the microscale situation. Our task is to develop equations that apply at the macroscale. Despite the difference in scales, the challenge of obtaining a general form is great. In the course of the subsequent development, we will indicate pitfalls and challenges. In this section we will consider some related investigations of interface and common curve movement. For subsequent convenience in comparing these results with those to be derived here, the notation used in original papers will be converted to that used in the TCAT formalism as possible.

Alts and Hutter [3] provided a continuum description of the dynamics and thermodynamics of the interface between ice and water. In that work, the conservation equations were developed at the microscale. These authors also recognized the need for an evolution equation to describe the change of the surface between the phases as a function of time and derived the equation

$$
\frac{\mathrm{D}(\mathrm{d} a)}{\mathrm{D} t}=\left(\nabla^{\prime} \cdot \mathbf{w}^{\prime}+J_{w} \mathbf{w} \cdot \mathbf{n}_{w}\right) \mathrm{d} a,
$$


where $\mathrm{D} / \mathrm{D} t$ is a time derivative following the surface, $\mathrm{d} a$ is a differential element of the surface, $\nabla^{\prime}$. is the surface divergence, $\mathbf{w}$ is the velocity of the surface, $\mathbf{w}^{\prime}$ is the portion of the surface velocity tangent to the surface, $\mathbf{n}_{w}$ is the unit vector normal to the water phase, and $J_{w}$ is the interfacial curvature equal to $\nabla^{\prime} \cdot \mathbf{n}_{w}$. This equation also was obtained in Moeckel [56]. Note that because this is a microscale form, the interaction of the edges of the surface with the common curve impact the solution of this equation through boundary conditions. Within a porous medium system, microscale eqn (1) would be averaged over the interfacial segments within an averaging volume to obtain the expression appropriate for the macroscale. For a multiphase system, equations such as eqn (1) must be averaged over each interface to obtain the set of equations for the interfaces. Because the ice-water system contains only two phases, a development of equations for common curves was not considered. Alts and Hutter [3] additionally provided microscale conservation equations for the interface properties that contribute to the complete microscale description of the ice water system.

Kalaydjian [41] provided an analysis of multiphase porous medium flow problems and included in his analysis the evolution of the fluid-fluid interface in space and time. His approach was based on the weight function averaging method developed by Marle [48,49] but supplemented with an equation to account for variations in the interfacial area between phases. Kalaydjian's perspective was at the macroscale with attention focused on a system consisting of a solid, water, and oil. Among the restrictions he imposed were the situation where the solid is completely wetted by a water phase of sufficient thickness to prevent interaction between the oil and the solid. This stipulation means that no common curve, which would form where three phases meet, exists. The interfaces were considered to be massless and the fluid-fluid interfaces were constrained to be spherical. The restriction to a massless interface was used to eliminate the need for a mass balance equation for the interface and to remove the inertial terms from the interfacial momentum equation. A relation between the interfacial area per volume between the phases, $\varepsilon^{w n}$, and the volume fraction of the oil phase, $\varepsilon^{n}$ was assumed to be of the form

$$
\frac{\partial \varepsilon^{w n}}{\partial t}+f \frac{J_{w}^{w n}}{2} \frac{\partial \varepsilon^{n}}{\partial t}=0
$$

where $J_{w}^{w n}$ is the curvature of the oil-water interface, and $f$ is a function postulated to depend on space, time, the deformation tensor of the interface, and $\varepsilon^{n}$. Based on this formulation, Kalaydjian [41] also obtained a dynamic equation for evolution of the capillary pressure as

$$
p^{n}-p^{w}+\gamma^{w n} f \frac{J_{w}^{w n}}{2}=L_{1} \frac{\partial \varepsilon^{n}}{\partial t},
$$

where $\gamma^{w n}$ is the interfacial tension, and $L_{1}$ is a model parameter. For a simple case where the fluid-fluid interface retains a spherical shape during displacement such that its deformation tensor is zero, Kalaydjian [41] indicates that $f=2$. In considering eqn (3), it is useful to note that, in fact, the capillary pressure is $p^{c}=-\gamma^{w n} J_{w}^{w n}$, the product of the interfacial tension and curvature. The deviation of $f$ from 2 and of the right side of eqn (3) from zero would indicate that the equilibrium condition $p^{c}=p^{n}-p^{w}$ has not been achieved.

Kalaydjian [41] also developed macroscale equations for the average of the interface velocity of the wetting-non-wetting interface, $\mathbf{v}^{w n}$. One of the equations presented is troublesome and is the expression 


$$
\gamma^{w n} \varepsilon^{w n}=L_{2} \nabla \cdot \mathrm{v}^{w n}
$$

where $L_{2}$ is a model parameter. The problem with this equation is that it requires that when the divergence of the average interface velocity is zero, either the interfacial tension or the interfacial area per volume must be zero. Thus use of this equation implies that either the interface will not exist or the interfacial tension will be zero at equilibrium. Clearly, this will not be the case in general, and thus this equation must be reevaluated. Since the equation was developed in the context of a full system analysis, an improved formulation could also impact other relations obtained.

In quite a different context, Ishii and Kim [37] have examined an area interface transport equation for the interfacial area between bubbles and a flowing fluid in the absence of a porous solid. The objective was to model co-current and countercurrent flows in pipes. Macroscale equations were developed based on the Boltzmann equation. The interfacial area transport equation proposed is

$$
\frac{\partial \varepsilon^{w n}}{\partial t}+\nabla \cdot\left(\varepsilon^{w n} \mathrm{v}^{w n}\right)-\frac{2}{3}\left(\frac{\varepsilon^{w n}}{\varepsilon^{n}}\right)\left[\frac{\partial \varepsilon^{n}}{\partial t}+\nabla \cdot\left(\varepsilon^{n} \mathrm{v}^{n}\right)-r^{\overline{\bar{n}}}\right]=r^{\overline{\overline{w n}}},
$$

where the $n$ phase is the bubbles, $r^{\bar{n}}$ is a rate of volume of $n$ phase generation by a nucleation source per unit volume of mixture, $r^{\overline{\overline{w n}}}$ is the source/sink rate for interfacial area due to coalescence and break-up of bubbles. The challenge identified for using this equation is the development of appropriate relations for the source and sink terms $[37,38,43]$. Inherently, the problem being considered is different from the porous medium case because one of the fluid phases is modeled as a collection of spherical bubbles and bubbles that transition into a cap-like geometry due to turbulent shear processes. Nevertheless, the equation formulated provides an interesting approach to capturing changes in the area between phases due to both expansion of the phase shape and generation processes due to coalescence and breakup. These latter two mechanisms can be considered as involving significant deformations from a given state, which are also expected to be important in multiphase porous medium systems.

There are several observations that can be made concerning eqn (5) that are important in distinguishing it from a porous medium area transport equation. First, the presence of a solid phase in a porous medium imposes some geometric constraints on the geometry of the phase distributions. The presence of a phase as a collection of spheres would be unlikely unless the spheres were very small. Second for a porous medium flow problem, parts of a phase can become entrapped in the solid medium, thus leading to drastically different behavior from the case of bubbles in a fluid. An instance in which this different behavior is important is in specification of the interface velocity, $\mathbf{v}^{w n}$. For the case where the $n$ phase is comprised of bubbles, Kim [43] makes the reasonable approximation that the velocity of the phase interface is approximately equal to the velocity of the disperse phase such that $\mathbf{v}^{w n} \approx \mathbf{v}^{n}$. For a porous medium system, this simplifying assumption might apply in the limit of small saturation of the $n$ phase such that it is essentially immobile. However, over the range of expected saturations, a more general approximation would be required for the interface velocity. Finally, no common curves exist for the two-phase fluid-bubble system, thus obviating the need for an equation describing common curve dynamics.

We can simplify eqn (5) for the uid-bubbles case employed by Kim [43] in which the condition was imposed that 


$$
\mathbf{v}^{w n}=\mathbf{v}^{n},
$$

to obtain

$$
\frac{\mathrm{D}^{w n} \varepsilon^{w n}}{\mathrm{D} t}+\frac{1}{3} \varepsilon^{w n} \mathbf{I}: \mathbf{d}^{w n}-\frac{2}{3}\left(\frac{\varepsilon^{w n}}{\varepsilon^{n}}\right) \frac{\mathrm{D}^{w n} \varepsilon^{n}}{\mathrm{D} t}=r^{\overline{w n}}-\frac{2}{3}\left(\frac{\varepsilon^{w n}}{\varepsilon^{n}}\right) r^{\overline{\bar{n}}},
$$

where with a slight abuse of notation, the rate of strain tensor in terms of an intrinsically averaged interfacial velocity is given as

$$
\mathbf{d}^{w n}=\frac{1}{2}\left[\nabla \mathbf{v}^{w n}+\left(\nabla \mathbf{v}^{w n}\right)^{\mathrm{T}}\right]
$$

When the $w n$ surface is the boundary of a sphere enclosing the $n$ phase, the identity holds that

$$
\varepsilon^{n} \nabla \varepsilon^{w n}-\frac{2}{3} \varepsilon^{w n} \nabla \varepsilon^{n}=0
$$

Incorporation of this relation into eqn (7) provides the simplification of the Ishii-Kim result to

$$
\frac{\partial \varepsilon^{w n}}{\partial t}+\frac{1}{3} \varepsilon^{w n} \mathbf{I}: \mathbf{d}^{w n}-\frac{2}{3}\left(\frac{\varepsilon^{w n}}{\varepsilon^{n}}\right) \frac{\partial \varepsilon^{n}}{\partial t}=r^{\overline{\overline{w n}}}-\frac{2}{3}\left(\frac{\varepsilon^{w n}}{\varepsilon^{n}}\right) r^{\overline{\bar{n}}}
$$

This equation will be discussed subsequently in comparison to the result derived here.

An approach based upon the use of change of scale averaging theorems to approximate an interfacial area transport equation for flow of two fluids in a porous medium system was advanced by Gray [21] and further developed by Gray et al. [30] using a less restrictive set of assumptions. Some approximations to the integrals in the mathematical averaging theorems that facilitate a change in scale were employed to obtain an evolution equation for interfacial area transport of the form [30] 


$$
\begin{aligned}
\frac{\mathrm{D}^{\bar{s}} \varepsilon^{w n}}{\mathrm{D} t} & \\
& -J_{w}^{w n} \frac{\mathrm{D}^{\bar{s}} \varepsilon^{w}}{\mathrm{D} t} \\
& -\cos \varphi^{\overline{\overline{w s, w n}}}\left(\varepsilon^{w s}+\varepsilon^{n s}\right) \frac{\mathrm{D}^{\bar{s}} \chi_{w s}^{s s}}{\mathrm{D} t} \\
& +\nabla \cdot\left(\varepsilon^{w n} \mathbf{G}^{w n}\right) \cdot\left(\mathbf{v}^{\overline{w n}}\right. \\
& \left.-\mathbf{v}^{\bar{s}}\right) \\
& +\varepsilon^{w n} \mathbf{G}^{w n}: \mathbf{d}^{\overline{\overline{w n}}} \\
& =-\left[\chi_{w s}^{s s} J_{w}^{w n}-\frac{\varepsilon^{w n s}}{\left(\varepsilon^{w s}+\varepsilon^{n s}\right)} \sin \varphi^{\overline{\overline{w s, w n}}}-\chi_{w s}^{s s}\left(J_{s}^{w s}-J_{s}^{s s}\right) \cos \varphi^{\overline{\overline{w s, w n}}}\right] \frac{\mathrm{D}^{\bar{s}} \varepsilon}{\mathrm{D} t} \\
& +\left[\nabla \cdot\left(\chi_{n s}^{s s} \varepsilon^{w s} \mathbf{G}^{w s}\right)+\varepsilon^{w s} \mathbf{G}^{w s} \cdot \nabla \chi_{w s}^{s s}\right] \cdot\left(\mathbf{v}^{\overline{w s}}\right. \\
& \left.-\mathbf{v}^{\bar{s}}\right) \cos \varphi^{\overline{w s, w n}} \\
& -\left[\nabla \cdot\left(\chi_{w s}^{s s} \varepsilon^{n s} \mathbf{G}^{n s}\right)-\varepsilon^{n s} \mathbf{G}^{n s} \cdot \nabla \chi_{w s}^{s s}\right] \cdot\left(\mathbf{v}^{\overline{n s}}\right. \\
& \left.-\mathbf{v}^{\bar{s}}\right) \cos \varphi^{\overline{w s, w n}} \\
& +\left(\chi_{n s}^{s s} \varepsilon^{w s} \mathbf{G}^{w s}: \mathbf{d}^{\overline{\overline{w s}}}-\chi_{w s}^{s s} \varepsilon^{n s} \mathbf{G}^{n s}: \mathbf{d}^{\overline{\overline{n s s}}}\right) \cos \varphi^{\overline{\overline{w s, w n}}},
\end{aligned}
$$

where the geometric orientation tensor for an interface $\imath$ separating an $\alpha$ and $\beta$ phase may be defined formally as [30]

$$
\mathbf{G}^{\iota}=\frac{1}{\varepsilon^{\iota} V} \int_{\Omega_{l}} \mathbf{n}_{\alpha} \mathbf{n}_{\alpha} \mathrm{dr}, \text { for } \iota \in \mathfrak{I}_{\mathrm{I}}, \alpha \in \mathfrak{I}_{\mathrm{P}}, \text { and } \Omega_{\iota}=\bar{\Omega}_{\alpha} \cap \bar{\Omega}_{\beta}
$$

the rate of strain tensor is

$$
\mathbf{d}^{\overline{\bar{i}}}=\frac{1}{2}\left[\nabla \mathbf{v}^{\bar{i}}+\left(\nabla \mathbf{v}^{\bar{\imath}}\right)^{\mathrm{T}}\right]
$$

$\varphi^{\overline{\overline{w s, w n}}}$ is the contact angle between the $w s$ and $w n$ interfaces; $\chi_{w s}^{s s}$ and $\chi_{n s}^{s s}$ are the fractions of the solid surface in contact with the $w$ and $n$ phases, respectively; $V$ is the averaging volume; $\mathbf{n}_{\alpha}$ is the outward unit normal vector from phase $\alpha$ at the boundary; $\Im_{P}$ is the index set of phases; $\Im_{I}$ is the index set of interfaces; $\Omega_{1}$ is the portion of the averaging domain occupied by the 1 entity; and $\Omega$ is the closure of a domain that includes the boundary. It is important to recognize that the single overbar in the superscripts on the velocities indicates that these quantities are mass-weighted average velocities. This type of velocity was employed because the area transport equation was derived from entropy inequality considerations. However, the velocities in eqn (11) should be simple averages, not mass-weighted averages, of their microscale counterparts, as will be addressed in a subsequent derivation. For purposes of this discussion, we will not consider this discrepancy in the employed definition of the velocity.

Let us simplify eqn (11) slightly by assuming that the curvature of the solid interface is not correlated to the fluid phase it contacts so that the difference between $J_{s}^{w s}$ and $J_{s}^{s s}$ is negligible. Also apply the product rule to the coefficients that multiply the differences 
between the fluid-solid interface velocities and the solid velocity. With these changes, eqn (11) becomes

$$
\begin{aligned}
\frac{\mathrm{D}^{\bar{s}} \varepsilon^{w n}}{\mathrm{D} t} & \\
& -J_{w}^{w n} \frac{\mathrm{D}^{\bar{s}} \varepsilon^{w}}{\mathrm{D} t} \\
& -\cos \varphi^{\overline{\overline{w s} w n}}\left(\varepsilon^{w s}+\varepsilon^{n s}\right) \frac{\mathrm{D}^{\bar{s}} \chi_{w s}^{s s}}{\mathrm{D} t} \\
& +\nabla \cdot\left(\varepsilon^{w n} \mathbf{G}^{w n}\right) \cdot\left(\mathrm{v}^{\overline{w n}}\right. \\
& \left.-\mathbf{v}^{\bar{s}}\right) \\
& +\varepsilon^{w n} \mathbf{G}^{w n}: \mathbf{d}^{\overline{\overline{w n}}} \\
& = \\
& -\left[\chi_{w s}^{s s} J_{w}^{w n}-\frac{\varepsilon^{w n s}}{\left(\varepsilon^{w s}+\varepsilon^{n s}\right)} \sin \varphi^{\overline{w s, w n}}\right] \frac{\mathrm{D}^{\bar{s}} \varepsilon}{\mathrm{D} t} \\
& +\left[\chi_{n s}^{s s} \nabla \cdot\left(\varepsilon^{w s} \mathbf{G}^{w s}\right)\right] \cdot\left(\mathbf{v}^{\overline{w s}}\right. \\
& \left.-\mathbf{v}^{\bar{s}}\right) \cos \varphi^{\overline{\overline{w s, w n}}} \\
& -\left[\chi_{w s}^{s s} \nabla \cdot\left(\varepsilon^{n s} \mathbf{G}^{n s}\right)\right] \cdot\left(\mathbf{v}^{\overline{n s}}\right. \\
& \left.-\mathbf{v}^{\bar{s}}\right) \cos \varphi^{\overline{\overline{w s, w n}}} \\
& +\left(\chi_{n s}^{s s} \varepsilon^{w s} \mathbf{G}^{w s}: \mathbf{d}^{\overline{\overline{w s}}}-\chi_{w s}^{s s} \varepsilon^{n s} \mathbf{G}^{n s}: \mathbf{d}^{\overline{\overline{n s}}}\right) \cos \varphi^{\overline{\overline{w s}, w n}} .
\end{aligned}
$$

For the purpose of comparison with other models, consider some simplifications to eqn (14). If the solid phase and solid surface dynamics are much slower than the dynamics of the fluid-fluid interface, terms involving $\mathrm{v}^{\overline{w s}}-\mathrm{v}^{\bar{s}}, \mathrm{v}^{\overline{n s}}-\mathrm{v}^{\bar{s}}, \mathbf{d}^{\overline{\overline{w s}}}$, and $\mathbf{d}^{\overline{\overline{n s}}}$ can be neglected. In this case, the right side of eqn (14) is approximately zero. The expression in [21] is recovered if the additional approximation is made that terms involving the velocity and rate of strain of the fluid-fluid interface are also considered negligible.

Eqn (14) has some features in common with the forms discussed previously. For example, if the solid-phase movement is negligible and all terms except the first two on the left side are neglected, eqn (14) reduces to the overly simple expression of eqn (2) with $f=2$.

When no solid phase is present, all the terms on the right hand side and the third term on the left hand side of the expression proposed in eqn (14) are zero and the remaining terms can be rearranged for comparison with eqn (10) as

$$
\frac{\partial \varepsilon^{w n}}{\partial t}+\varepsilon^{w n} \mathbf{G}^{w n}: \mathbf{d}^{\overline{\overline{w n}}}+J_{w}^{w n} \frac{\partial \varepsilon^{n}}{\partial t}=-\nabla \cdot\left(\varepsilon^{w n} \mathbf{G}^{w n}\right) \cdot \mathbf{v}^{\overline{w n}},
$$

where use has been made of the fact that $\varepsilon^{w}+\varepsilon^{n}=1$ in the absence of a solid phase. When the $n$ phase has no preferred orientation, as with spherical bubbles or randomly oriented elements of this phase, $\mathbf{G}=\mathbf{I} / 3$ and eqn (15), which is rooted in considerations from the entropy inequality and averaging theorems, becomes

$$
\frac{\partial \varepsilon^{w n}}{\partial t}+\frac{1}{3} \varepsilon^{w n} \mathbf{1}: \mathbf{d}^{\overline{\overline{w n}}}+J_{w}^{w n} \frac{\partial \varepsilon^{n}}{\partial t}=-\nabla \cdot\left(\varepsilon^{w n} \mathbf{G}^{w n}\right) \cdot \mathbf{v}^{\overline{w n}} .
$$


Comparison of eqn (16) with eqn (10) shows a degree of consistency between the two forms when the difference between density-weighted and unweighted averaging is unimportant. When the $n$ phase is composed of bubbles, the curvature of the $w n$ interface satisfies the equality $J_{w}^{w n}=-2 \varepsilon^{w n} /\left(3 \varepsilon^{n}\right)$. The term on the right side of eqn (16) to some degree, at least, can be seen in conjunction with the first term on the left to form a material derivative of interfacial surface area. A corresponding term does not appear in the form of the Ishii-Kim equation obtained here as eqn (10). On the other hand, no term was included in eqn (14) to account for coalescence or breakup. Despite these differences, in consideration of the very different systems for which they were intended to apply, the similarity between the equations under restricted conditions is encouraging. Additionally, we note that the first two terms of eqn (16) are the same as the terms in the form employed in eqn (2).

We emphasize the fact that evolution equations for specific interfacial area and common curve length are not conservation equations, since areas and lengths are not strictly conserved quantities. Therefore, such evolution equations are not consequences of mass conservation. An area evolution equation and a mass conservation equation do not contradict each other; they are independent expressions. In recent work, some specific interfacial area equations have been proposed that suggest interface equations are, indeed, consequences of mass conservation [57-60]. Although this approach can be questioned on conceptual grounds, it nevertheless provides an equation that appears to be somewhat similar to the expression developed based upon the averaging theorem approach. For this reason, a careful analysis of this mass conservation approach will be considered.

First we note that for an entity in a multiphase porous medium system, the macroscale mass conservation equations for phases, interphases, and common curves $[23,39]$ can be expressed as

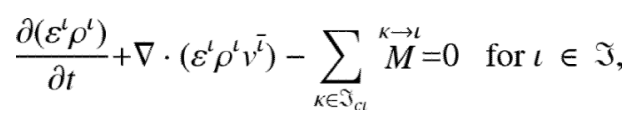

where $\rho^{l}$ is mass density of the $\imath$ entity, ${ }_{M}^{M}$ is the rate of mass exchange from the $\kappa$ entity to the $r$ entity, $\Im$ is the index set containing indexes for all phases, interfaces, and the common curve in a two-fluid-phase porous medium system, $\imath$ is a particular index of interest, and $\Im_{c l}$ is the index set of entities connected to entity $\imath$ that can exchange mass with the particular entity . Summation of this expression over all the entities cancels out the mass exchange terms resulting in

$$
\sum_{t \in \mathfrak{Y}}\left[\frac{\partial\left(\varepsilon^{t} \rho^{t}\right)}{\partial t}+\nabla \cdot\left(\varepsilon^{t} \rho^{t} \mathbf{v}^{\bar{c}}\right)\right]=0 .
$$

The terms in this equation related to the $w n$ interface can be isolated from the summation to obtain

$$
\frac{\partial\left(\varepsilon^{w n} \rho^{w n}\right)}{\partial t}+\nabla \cdot\left(\varepsilon^{w n} \rho^{w n} \mathbf{v}^{\overline{w n}}\right)=-\sum_{t \in\{\mathfrak{\Im}-w n\}}\left[\frac{\partial\left(\varepsilon^{l} \rho^{l}\right)}{\partial t}+\nabla \cdot\left(\varepsilon^{\imath} \rho^{t} \mathbf{v}^{\bar{t}}\right)\right] .
$$

In the work of Niessner and Hassanizadeh [57-60], the stipulation is made that $\rho^{w n}$ must be non-zero and constant. Both of these requirements are restrictive and, indeed, should not be fundamental in describing interface dynamics. We do note, however, that if the mass density 
of an interface were zero, all terms involving that interface would be eliminated from the mass balance eqn (18). In that circumstance an attempt to produce an equation for massless interface dynamics from an equation for the mass conservation of that interface would not be possible. Nevertheless, with the restriction of a non-zero, constant density interface imposed to facilitate investigation of a possible interfacial area equation based on mass conservation, division by the constant interface density $\rho^{w n}$ yields

$$
\frac{\partial \varepsilon^{w n}}{\partial t}+\nabla \cdot\left(\varepsilon^{w n} \mathbf{v}^{w n}\right)=-\frac{1}{\rho^{w n}} \sum_{t \in\{\mathfrak{Y}-w n\}}\left[\frac{\partial\left(\varepsilon^{l} \rho^{l}\right)}{\partial t}+\nabla \cdot\left(\varepsilon^{l} \rho^{l} \mathbf{v}^{\bar{l}}\right)\right]
$$

where the mass average velocity of the material in the $w n$ interface, $\mathbf{v}^{\overline{w n}}$, is equal to the spatially averaged velocity, $\mathbf{v}^{w n}$, since $\rho^{w n}$ has been deemed to be constant. In [57-60], an assumption is employed that the $w n$ area transport equation based on mass conservation with constant, non-zero $\rho^{w n}$ is

$$
\frac{\partial \varepsilon^{w n}}{\partial t}+\nabla \cdot\left(\varepsilon^{w n} \mathbf{v}^{w n}\right)=\widehat{e}^{w n} \frac{\partial s^{w}}{\partial t},
$$

where $\hat{e}^{w n}$ is considered to be a function only of saturation, $s^{w}$, and capillary pressure, $p^{c}$. This assumption is tantamount to specifying that the right side of eqn (20) can be approximated as the right side of eqn (21). The presumption that $\hat{e}^{w n}$ depends on capillary pressure is unnecessary given that none of the quantities on the right side of the system mass conservation expression eqn (20), either independently or in combination with other variables, requires the existence of capillary pressure. In summary, eqn (21) relies on the limiting requirements that $\rho^{w n}$ be constant and non-zero, that $\hat{e}^{w n}$ depends on capillary pressure, and that the proposed right side of the equation properly accounts for system dynamics. We will investigate whether eqn (21) is a reasonable hypothesis capable of describing evolution of the fluid-fluid interfacial area following the derivation of the evolution equation based on averaging theorems.

Niessner and Hassanizadeh recognize that there is a need for an equation that describes the velocity of the $w n$ interface. The form selected is [57-60]

$$
\mathbf{v}^{w n}=-\widehat{\mathbf{K}}^{w n} \cdot \nabla \varepsilon^{w n},
$$

where $\hat{\mathbf{K}}^{w n}$ is termed an interfacial permeability tensor. It can be seen from eqn (22) that, unless $\hat{\mathbf{K}}^{w n}$ goes to zero when the interfacial velocity is zero, the interfacial area density must be constant at equilibrium. In the same way that saturation need not be constant at equilibrium, it is likewise implausible that the density of the area between the fluid phases should be constant. Thus, eqn (22) is restrictive such that predictions made based on this equation could be non-physical. Although a reasonable hypothesis was made for the interface velocity when it is the boundary of a bubble, eqn (6), for the more complex case of two fluid phases within a porous medium, eqns (4) and (22) are restrictive such that they must be replaced on physical grounds.

The main point of this section is to illustrate the complexities involved in modeling the dynamics of the interface between fluid phases in multiphase porous medium systems. We emphasize the importance of treating the equation for interface dynamics as being related to differential geometry and as being completely distinct from conservation equations, in particular from the equation of mass conservation for the interface. Additionally, the need to 
ensure that proposals for the interface velocity be carefully considered to ensure they do not predict unphysical behavior.

\section{Averaging Theorems and Geometric Parameters}

In the subsequent derivations in this paper, useful forms of interface and common curve evolution equations will be obtained from averaging theorems. In this section, the averaging theorems will be supplied and rearranged to forms particularly useful for the task at hand. The theorems for phases are found in many references ([e.g., $\left.\left.{ }^{4}, 6,24,74,85\right]\right)$ while the averaging theorems for interfaces [22] and common curves [29] are also of importance for the present development. All the theorems of interest are collected in [29], so that document is used as the source for the individual theorems employed here. Additionally some quantities will be defined in terms of averaging operators. These quantities arise in the averaging theorems. In this section, no approximations will be employed, beyond the scale considerations that gird the averaging theorems.

The objective here is to provide the mathematical framework that can be exploited subsequently using appropriate approximations to obtain the interface and common curve evolution equations.

For derivation of the interface dynamics, the averaging theorems are applied to constant scalars and vectors. These averaging theorems are expressed using an averaging operator defined as

$$
\left\langle\mathscr{P}_{i}\right\rangle_{\Omega_{j}, \Omega_{k}, W}=\frac{\int_{\Omega_{j}} W \mathscr{P}_{i} d \mathrm{r}}{\int_{\Omega_{k}} W d \mathrm{r}},
$$

where $\mathscr{P}_{i}$ is a microscale quantity to be averaged, $\Omega_{j}$ and $\Omega_{k}$ are domains of integration, and $W$ is a weighting function. If $W$ is not specified, it is assumed to be 1 .

The gradient theorem for relating the average of the gradient of a microscale phase property to the gradient of an average property plus a boundary term is [29]

Theorem $1(G[3,(3,0), 0])$

$$
\left\langle\nabla f_{i}\right\rangle_{\Omega_{l}, \Omega}=\nabla\left\langle f_{i}\right\rangle_{\Omega_{l}, \Omega}+\sum_{\kappa \in \Im_{c l}}\left\langle\mathbf{n}_{\iota} f_{i}\right\rangle_{\Omega_{k}, \Omega}, \quad \text { for } \iota \in \mathfrak{J}_{\mathrm{P}}
$$

where the microscale scalar function $f_{l}$ is defined, continuous, and differentiable in $\Omega_{l} ; \Omega_{l}$ is the portion of the total domain, $\Omega$, occupied by the t phase; $\mathbf{n}_{t}$ is a unit outward normal vector from the boundary of $\Omega_{l} ; \Im_{c l}$ is the index set of connected entities, which consists of a set of interfaces formed at the intersection of the t phase and other phases in the system; and $\Im_{\mathrm{P}}$ is the index set of phases.

The transport theorem for relating the average partial time derivative of a microscale phase property to the partial derivative of the macroscale average plus a boundary term is [29]

Theorem $2(T[3,(3,0), 0])$ 


$$
\left\langle\frac{\partial f_{l}}{\partial t}\right\rangle_{\Omega_{l}, \Omega}=\frac{\partial}{\partial t}\left\langle f_{i}\right\rangle_{\Omega_{i}, \Omega}-\sum_{\kappa \in \mathfrak{I}_{a l}}\left\langle n_{l} \cdot w_{\kappa} f_{\imath}\right\rangle_{\Omega_{\kappa}, \Omega}, \text { for } \iota \in \mathfrak{J}_{\mathrm{P}},
$$

where $f_{l}$ is continuous and differentiable in $\Omega_{l}$ and time; $t$ is time; $\Omega_{l}$ is the portion of the total domain, $\Omega$, occupied by the i phase; $\mathbf{n}_{1}$ is a unit outward normal vector from the boundary of $\Omega_{l} ; \mathbf{w}_{\kappa}$ is the velocity vector for the $\kappa$ interface; $\widetilde{I}_{c l}$ is the index set of connected entities, which consists of a set of interfaces formed at the intersection of the 1 phase and other phases in the system; and $\mathfrak{\Im}_{\mathrm{P}}$ is the index set of phases.

When $f_{\mathrm{l}}$ is a constant, eqn (24) provides an expression for the gradient of the volume fraction of the phase as

$$
\nabla \varepsilon^{\iota}=-\sum_{\kappa \in \mathfrak{I}_{c l}}\left\langle n_{l}\right\rangle_{\Omega_{\kappa}, \Omega} \text { for } \iota \in \mathfrak{J}_{\mathrm{p}}
$$

With $f_{1}$ a constant, eqn (25) provides an expression for the time derivative of the volume fraction of the phase as

$$
\frac{\partial \varepsilon^{\iota}}{\partial t}=\sum_{\kappa \in \mathfrak{I}_{c l}}\left\langle\mathbf{n}_{\iota} \cdot \mathbf{w}_{\kappa}\right\rangle_{\Omega_{\kappa}, \Omega} \quad \text { for } \iota \in \mathfrak{J}_{\mathrm{P}}
$$

In these last two equations, $\Omega_{\kappa}$ is the surface region bounding the phase. Therefore, these theorems will prove useful in relating area changes to phase dynamics.

The gradient theorem for averaging a microscale interface quantity to the macroscale relates the average of the surface gradient of a microscale property to the spatial gradient of the average plus terms that account for the surface shape boundary processes. This equation is [29]

\section{Theorem $3(\mathrm{G}[2,(3,0), 0])$}

$$
\left\langle\nabla^{\prime} f_{l}\right\rangle_{\Omega_{l}, \Omega}=\nabla\left\langle f_{l}\right\rangle_{\Omega_{l}, \Omega}-\nabla \cdot\left\langle\mathbf{n}_{\kappa} \mathbf{n}_{\kappa} f\right\rangle_{\Omega_{l}, \Omega}+\left\langle\left(\nabla^{\prime} \cdot \mathbf{n}_{k}\right) \mathbf{n}_{\kappa} f_{l}\right\rangle_{\Omega_{l}, \Omega^{2}}+\sum_{\gamma \in\left(\Im_{c l} \cap \Im_{C}\right)}\left\langle\mathbf{n}_{l} f_{l}\right\rangle_{\Omega_{\gamma}, \Omega} \text {, for } \iota \in \mathfrak{J}_{I},
$$

where the microscale gradient operator restricted to an interface is $\nabla^{\prime}=\nabla-\mathbf{n}_{\kappa} \mathbf{n}_{\kappa} \cdot \nabla ;$ the microscale scalar function $f_{l}$ is defined, continuous and differentiable in $\Omega_{l}$; $\Omega_{l}=\Omega_{\kappa} \cap \bar{\Omega}_{\beta}$ is the interface entity in domain $\Omega$ formed at the intersection of two closed phase domains denoted $\bar{\Omega}_{\kappa}$ and $\bar{\Omega}_{\beta}$ with $\kappa, \beta \in \Im_{\mathrm{P}} ; \mathbf{n}_{1}$ is a unit vector tangent to $\Omega_{l}$ and orthogonal to its bounding common curve; $\mathbf{n}_{\mathrm{\kappa}}$ is a unit vector normal to the $\mathrm{l}$ interface and outward normal to the $\kappa$ phase; $\widetilde{I}_{c l}$ is the index set of connected entities that consists of a set of bounding phases and common curves; $\Im_{\mathrm{P}}$ is the index set of phases; $\Im_{\mathrm{I}}$ is the index set of interfaces; and $\Im_{\mathrm{C}}$ is the index set of common curves.

The surface transport theorem for averaging a microscale quantity to the macroscale relates the average of the partial time derivative fixed to the surface to the time derivative of the macroscale average plus effects due to deformation of the surface and changes in its size due to boundary motion. This theorem is [29] 
Theorem $4(T[2,(3,0), 0])$

$\left\langle\frac{\partial^{\prime} f_{\iota}}{\partial t}\right\rangle_{\Omega_{l}, \Omega}=\frac{\partial}{\partial t}\left\langle f_{\iota}\right\rangle_{\Omega_{l}, \Omega}+\nabla \cdot\left\langle\mathbf{n}_{\kappa} \mathbf{n}_{\kappa} \cdot \mathbf{w}_{\iota} f_{\iota}\right\rangle_{\Omega_{l}, \Omega}-\left\langle\left(\nabla^{\prime} \cdot \mathbf{n}_{\kappa}\right) \mathbf{n}_{k} \cdot \mathbf{w}_{\iota} f_{\iota}\right\rangle_{\Omega_{l}, \Omega}-\sum_{\gamma \in\left(\mathfrak{\Im}_{c l} \cap \Im_{C}\right)}\left\langle\mathbf{n}_{\iota} \cdot \mathbf{w}_{\gamma} f_{l}\right\rangle_{\Omega \gamma, \Omega}$, for $\iota \in \mathfrak{I}_{\mathrm{I}}$,

where the microscale time derivative restricted to an interface is

$$
\frac{\partial^{\prime}}{\partial t}=\frac{\partial}{\partial t}+\mathbf{w}_{\iota} \cdot \mathbf{n}_{\kappa} \mathbf{n}_{k} \cdot \nabla
$$

the microscale gradient operator restricted to an interface is $\nabla^{\prime}=\nabla-\mathbf{n}_{\mathrm{K}} \mathbf{n}_{\mathrm{K}} \cdot \nabla$; the microscale scalar function $f_{l}$ is defined, continuous and differentiable in $\Omega_{l}$ and time; $\Omega_{l}=$ $\Omega_{\kappa} \cap \Omega_{\beta}$ is the interface entity in domain $\Omega$ formed at the intersection of two closed phase domains denoted $\overline{\Omega_{\kappa}}$ and $\overline{\Omega_{\beta}}$ with $\kappa, \beta \in \widetilde{\Im}_{\mathrm{P}} ; \mathrm{w}_{1}$ is the velocity of the $\mathrm{l}$ interface; $\mathbf{n}_{\mathbf{l}}$ is a unit vector tangent to $\Omega_{l}$ and orthogonal to the bounding common curve; $\mathbf{n}_{\mathrm{\kappa}}$ is a unit vector normal to the $\mathrm{i}$ interface and outward normal to the $\kappa$ phase; $\mathfrak{I}_{C l}$ is the index set of connected entities that consists of a set of bounding phases and common curves; $\Im_{P}$ is the index set of phases; $\Im_{I}$ is the index set of interfaces; and $\Im_{C}$ is the index set of common curves.

For a constant microscale property, $f_{\mathfrak{l}}$, at an interface, eqn (28) provides an expression for the gradient of the interfacial area density. Similarly, for constant $f_{l}$, a relation for the time derivative of the interfacial area density is obtained from eqn (29). The equation for the gradient of area density for the two-fluid-phase system of interest, with only a wns common curve, is

$$
0=\nabla \varepsilon^{\iota}-\nabla \cdot\left\langle\mathbf{n}_{\kappa} \mathbf{n}_{\kappa}\right\rangle_{\Omega_{1}, \Omega}+\left\langle\left(\nabla^{\prime} \cdot \mathbf{n}_{\kappa}\right) \mathbf{n}_{\kappa}\right\rangle_{\Omega_{1}, \Omega}+\left\langle\mathbf{n}_{\iota}\right\rangle_{\Omega_{w n s}, \Omega} \text { for } \iota \in \mathfrak{I}_{\mathrm{I}}
$$

The temporal derivative of area density is related to the movement of the surface and the common curve according to

$0=\frac{\partial \varepsilon^{\iota}}{\partial t}+\nabla \cdot\left\langle\mathbf{n}_{\kappa} \mathbf{n}_{K} \cdot \mathbf{w}_{\iota}\right\rangle_{\Omega_{t}, \Omega}-\left\langle\left(\nabla^{\prime} \cdot \mathbf{n}_{K}\right) \mathbf{n}_{\kappa} \cdot \mathbf{w}_{\iota}\right\rangle_{\Omega_{t}, \Omega}-\left\langle\mathbf{n}_{\iota} \cdot \mathbf{w}_{w n s}\right\rangle_{\Omega_{w n s}, \Omega}$ for $\iota \in \mathfrak{J}_{I}$.

It is revealing that in these equations, the only velocities that impact the change in the area density are the normal components of the velocity of the surface and the velocity of the common curve bounding the surface in the direction tangent to the surface. The first of these accounts for stretching due to deformation of the surface while the latter accounts for an increase in size due to movement of the edges. Tangential velocity of the surface does not change its area density, although non-uniform velocity of the common curve bounding the surface in a direction tangent to the surface and normal to the edge does involve a change in surface area.

Terms appear in eqns (31) and (32) that suggest a useful definition of macroscale quantities. First is the geometric orientation tensor for an interface encountered previously in eqn (12). In the interest of a self-contained development, we repeat the definition here as

$$
\mathbf{G}^{\iota}=\left\langle\mathbf{n}_{\kappa} \mathbf{n}_{\kappa}\right\rangle_{\Omega_{l}, \Omega_{l}} \text { for } \iota \in \mathcal{J}_{\mathrm{I}}
$$


This quantity is a measure of the isotropy of the orientation of an interface type and has the properties that it is symmetric and the sum of its diagonals equals 1 . When the interface 1 between phases is randomly oriented within an REV, $\mathbf{G}^{\mathbf{l}}=\mathbf{I} / 3$ [30]. The second quantity of interest is the macroscale average of the sum of the principal curvatures, $J_{K}^{t}$ defined as

$$
J_{\kappa}^{\iota}=\left\langle\nabla^{\prime} \cdot \mathbf{n}_{\kappa}\right\rangle_{\Omega_{t}, \Omega_{t}} \text { for } \iota \in \mathfrak{I}_{\mathrm{I}},
$$

where $J_{\kappa}^{i}$ is the macroscale curvature of the $\mathrm{i}$ interface with respect to the normal direction to the interface determined by the vector $\mathbf{n}_{\kappa}$ that is outward directed from the $\kappa$ phase at the interface. Both $\mathbf{G}^{\mathbf{l}}$ and $\boldsymbol{J}_{\kappa}^{\iota}$ will be employed for the $w s, n s$, and $w n$ interfaces.

The solid surface in this system will be considered to be smooth such that at a common curve anywhere on the surface

$$
\mathbf{n}_{w s}=-\mathbf{n}_{n s}
$$

where $\mathbf{n}_{w s}$ and $\mathbf{n}_{n s}$ are unit vectors tangent to the respective surfaces that point outward from the respective surface and are normal to the bounding wns common curve.

At the common curve, the unit vector $\mathbf{n}_{w n}$ may be decomposed into a part that is tangent to the solid surface and a part that is normal to the solid according to

$$
\mathbf{n}_{w n}=\mathbf{n}_{w s} \cos \varphi_{w s, w n}-\mathbf{n}_{s} \sin \varphi_{w s, w n},
$$

where $\varphi_{w s, w n}$ is the contact angle that spans the $w$ phase between the $w s$ and $w n$ surfaces. The macroscale estimate of the cos of the contact angle is defined as [39]

$$
\cos \varphi^{\overline{\overline{w s, w n}}}=\frac{\left\langle\cos \left(\varphi_{w s, w n}\right)\right\rangle_{\Omega_{w n s}, \Omega_{w n s}}}{\left[\left\langle\cos \left(\varphi_{w s, w n}\right)\right\rangle_{\Omega_{w n s}, \Omega_{w n s}}^{2}+\left\langle\sin \left(\varphi_{w s, w n}\right)\right\rangle_{\Omega_{w n s}, \Omega_{w n s}}^{2}\right]^{1 / 2}}
$$

or, equivalently

$$
\sin \varphi^{\overline{\overline{w s, w n}}}=\frac{\left\langle\sin \left(\varphi_{w s, w n}\right)\right\rangle_{\Omega_{w n s}, \Omega_{w n s}}}{\left[\left\langle\cos \left(\varphi_{w s, w n}\right)\right\rangle_{\Omega_{w n s}, \Omega_{w n s}}^{2}+\left\langle\sin \left(\varphi_{w s, w n}\right)\right\rangle_{\Omega_{w n s}, \Omega_{w n s}}^{2}\right]^{1 / 2}}
$$

The gradient theorem for averaging a microscale common curve quantity to the macroscale relates the average of the gradient along the curve of a microscale property to the spatial gradient of a macroscale property plus terms that account for changes due to deformation of the curve and processes at the end of the curve. This theorem is [29]

Theorem $5(G[1,(3,0), 0])$

$\left\langle\nabla^{\prime \prime} f_{\imath}\right\rangle_{\Omega_{l}, \Omega}=\nabla \cdot\left\langle\mathbf{n}_{\iota} \mathbf{n}_{t} f_{\iota}\right\rangle_{\Omega_{l}, \Omega}-\left\langle\mathbf{n}_{t} \cdot \nabla^{\prime \prime} \mathbf{n}_{\imath} f_{\iota}\right\rangle_{\Omega_{l}, \Omega}+\left.\frac{1}{V} \sum_{j \in \mathfrak{J}_{\mathrm{Pu}}} \mathbf{e}_{\imath} f_{\iota}\right|_{\Omega_{j}}, \quad$ for $\iota \in \mathfrak{J}_{\mathrm{C}}$, 
where the microscale gradient operator restricted to a common curve is $\nabla^{\prime \prime}=\mathbf{n}_{1} \mathbf{n}_{1} \cdot \nabla ;$ the microscale scalar function $f_{l}$ is defined, continuous and differentiable in $\Omega_{l} ; \Omega_{l}=\bar{\Omega}_{\beta} \cap \bar{\Omega}_{\gamma} \cap$ $\Omega_{\delta}$ is the common curve entity in the domain $\Omega$ formed at the intersection of three closed interfaces $\overline{\Omega_{\beta}}, \overline{\Omega_{\gamma}}$, and $\overline{\Omega_{\delta}}$ with $\beta, \gamma, \delta \in \Im_{\mathbb{I}} ; \mathbf{n}_{1}$ is a unit vector tangent to the common curve $\Omega_{l} ; V$ is the volume of the averaging region; $\mathbf{e}_{1}$ is a unit vector normal to the endpoint of the common curve and positive outward; $\Im_{I}$ is the index set of interfaces; $\Im_{C}$ is the index set of common curves; and $\Im_{\mathrm{Pt}}$ is the index set of common points for common curve $\mathrm{t}$.

The transport theorem for averaging a microscale common curve quantity to the macroscale is [29]

Theorem 6 (T[1,(3,0),0])

$$
\left\langle\frac{\partial^{\prime \prime} f_{\iota}}{\partial t}\right\rangle_{\Omega_{l}, \Omega}=\frac{\partial}{\partial t}\left\langle f_{\iota}\right\rangle_{\Omega_{l}, \Omega}+\nabla \cdot\left\langle\left(\mathbf{I}-\mathbf{n}_{\iota} \mathbf{n}_{\iota}\right) \cdot \mathbf{w}_{\iota} f_{\iota}\right\rangle_{\Omega_{\imath}, \Omega}+\left\langle\mathbf{n}_{\iota} \cdot \nabla^{\prime \prime} \mathbf{n}_{\iota} \cdot \mathbf{w}_{\iota} f_{\iota}\right\rangle_{\Omega_{l}, \Omega}-\left.\frac{1}{V} \sum_{j \in \mathfrak{Y}_{\mathrm{Pu}}} \mathbf{e}_{\iota} \cdot \mathbf{w}_{j} f_{\iota}\right|_{\Omega_{j}}, \quad \text { for } \iota \in \mathfrak{J}_{\mathrm{C}}
$$

where the microscale time derivative restricted to the common curve is

$$
\frac{\partial^{\prime \prime}}{\partial t}=\frac{\partial}{\partial t}+\mathbf{w}_{\iota} \cdot\left(I-\mathbf{n}_{\iota} \mathbf{n}_{l}\right) \cdot \nabla
$$

the microscale gradient operator restricted to a common curve is $\nabla^{\prime \prime}=\mathbf{n}_{1} \mathbf{n}_{1} \cdot \nabla$; the microscale scalar function $f_{l}$ is defined, continuous and differentiable in $\Omega_{l}$ and time; $\Omega_{l}=$ $\overline{\Omega_{\beta}} \cap \overline{\Omega_{\gamma}} \cap \overline{\Omega_{\delta}}$ is the common curve entity in the domain $\Omega$ formed at the intersection of three closed interfaces $\overline{\Omega_{\beta}}, \overline{\Omega_{\gamma}}$, and $\overline{\Omega_{\delta}}$ with $\beta, \gamma \delta \in \Im_{\mathrm{I}} ; \mathbf{w}_{1}$ is the velocity of the common curve; $\mathbf{n}_{1}$ is a unit vector tangent to the common curve $\Omega_{l} ; V$ is the volume of the averaging region; $\mathbf{e}_{\mathbf{t}}$ is a unit vector normal to the endpoint of the common curve and positive outward; $\Im_{I}$ is the index set of interfaces; $\Im_{\mathrm{C}}$ is the index set of common curves; and $\Im_{\mathrm{Pt}}$ is the index set of common points for common curve $t$.

Now we will find the forms of these theorems when the microscale property, $f_{t}$, is constant and no common points exist, as with a two-fluid phase porous medium system. Eqn (39) provides an expression for the gradient of the common curve length per volume, with no common points, as

$$
\nabla \varepsilon^{w n s}=\nabla \cdot\left\langle\left(\mathbf{I}-\mathbf{n}_{w n s} \mathbf{n}_{w n s}\right)\right\rangle_{\Omega_{w n s}, \Omega}+\left\langle\left(\mathbf{n}_{w n s} \cdot \nabla^{\prime \prime} \mathbf{n}_{w n s}\right)\right\rangle_{\Omega_{w n s}, \Omega} .
$$

Eqn (40) gives an expression for the time derivative of the common curve density in the form

$$
\frac{\partial \varepsilon^{w n s}}{\partial t}=-\nabla \cdot\left\langle\left(\mathbf{I}-\mathbf{n}_{w n s} \mathbf{n}_{w n s}\right) \cdot \mathbf{w}_{w n s}\right\rangle_{\Omega_{w n s}, \Omega}-\left\langle\left(\mathbf{n}_{w n s} \cdot \nabla^{\prime \prime} \mathbf{n}_{w n s}\right) \cdot \mathbf{w}_{w n s}\right\rangle_{\Omega_{w n s}, \Omega}
$$

In eqns (42) and (43), the only velocity that appears is that which is orthogonal to the common curve. This is consistent with the expectation that the movement of the curve in a direction tangent to itself does not alter the amount of the curve in the averaging volume. However, movement orthogonal to the curve causes deformations and stretching that changes the common curve length per volume. Because no common points exist that would 
mark the ends of the common curve, there is no lengthening of the curve due to the movement of its ends.

Our objective is to make use of eqns (42) and (43) to develop a single evolution equation for the common curve. In these two equations, a couple of terms appear that are precursors to useful macroscale quantities. Define the common curve orientation tensor, $\mathbf{G}^{\text {wns }}$, as

$$
\mathbf{G}^{w n s}=\left\langle\left(\mathbf{I}-\mathbf{n}_{w n s} \mathbf{n}_{w n s}\right)\right\rangle_{\Omega_{w n s}, \Omega_{w n s}} .
$$

When the orientation of the common curve has no preferred direction, $\mathbf{G}^{\text {wns }}=2 \mathbf{I} / 3$ [30]. This orientation tensor is similar to the orientation tensor employed in modeling flowinduced fiber orentation [11,31]. The vector $\mathbf{n}_{w n s} \cdot \nabla " \mathbf{n}_{w n s}$ is normal to $\mathbf{n}_{w n s}$ and may be written in terms of the geodesic curvature, $\kappa_{G w n s}$, and the normal curvature, $\kappa_{N w n s}$, as

$$
\mathbf{n}_{w n s} \cdot \nabla^{\prime \prime} \mathbf{n}_{w n s}=\kappa_{G w n s} \mathbf{n}_{w s}+\kappa_{N w n s} \mathbf{n}_{s},
$$

where

$$
\kappa_{G w n s}=\mathbf{n}_{w n s} \cdot \nabla^{\prime \prime} \mathbf{n}_{w n s} \cdot \mathbf{n}_{w s}
$$

and

$$
\kappa_{N w n s}=\mathbf{n}_{w n s} \cdot \nabla^{\prime \prime} \mathbf{n}_{w n s} \cdot \mathbf{n}_{s}
$$

Then the macroscale geodesic and normal curvature may be defined, respectively, as averages of their microscale counterparts such that

$$
\kappa_{G}^{w n s}=\left\langle\kappa_{G w n s}\right\rangle_{\Omega_{w n s}, \Omega_{w n s}}=\left\langle\mathbf{n}_{w n s} \cdot \nabla^{\prime \prime} \mathbf{n}_{w n s} \cdot \mathbf{n}_{w s}\right\rangle_{\Omega_{w n s}, \Omega_{w n s}},
$$

and

$$
\kappa_{N}^{w n s}=\left\langle\kappa_{N w n s}\right\rangle_{\Omega_{w n s}, \Omega_{w n s}}=\left\langle\mathbf{n}_{w n s} \cdot \nabla^{\prime \prime} \mathbf{n}_{w n s} \cdot \mathbf{n}_{s}\right\rangle_{\Omega_{w n s}, \Omega_{w n s}}
$$

The orientation tensor for the curve and its normal and geodesic curvatures play important roles in the derivation of the evolution equation for the specific common curve length.

\section{Interface Evolution}

In this section, the averaging theorems will be employed to develop evolution equations for the interfaces between the solid and fluid phases and for the evolution of the fluid-fluid interface. The study is restricted to consideration of a system composed of a single solid, denoted as the $s$ phase, and two fluid phases, with the wetting phase denoted as the $w$ phase and the non-wetting phase denoted as the $n$ phase. The equations that will be obtained will rely on some judicious approximations applied to the theorems and definitions of the last section. We point out that the equations for evolution of the interfaces between phases will be developed based solely on the mathematics of the averaging theorems for phases, interfaces, and common curves and do not rely on conservation equations of mass, momentum, or energy. In the derivation here, we will be combining divergence and 
transport averaging theorems to obtain a single evolution equation. Since these equations are independent, the combining will be done making use of an initially unspecified macroscale velocity denoted as $\mathbf{W}^{\bar{\alpha}}$, where the superscript indicates this is a macroscale velocity associated with some entity $\alpha$. The double overbar indicates that the averaging process used to derive $\mathbf{W}^{\bar{\alpha}}$ from the microscale will have to be specified. After the theorems have been combined, a convenient constitutive selection will be made for the velocity. This approach shares some similarities with Kim [43] who developed a general equation that involved the velocity of a bubble surface. Then, he made use of a constitutive assumption that this velocity was equal to the velocity of the fluid in the bubble.

The dot product of eqn (26) with the arbitrary macroscale velocity, $\mathbf{W}^{\overline{\bar{\alpha}}}$, and addition to eqn (27) provides the material derivative of the volume fraction of phase 1 as

$$
\frac{\mathrm{D}^{\overline{\bar{\alpha}}} \varepsilon^{\iota}}{\mathrm{D} t}=\sum_{\kappa \in \mathfrak{I}_{\mathrm{c} \iota}}\left\langle\mathbf{n}_{\iota} \cdot\left(\mathbf{w}_{\kappa}-\mathbf{W}^{\overline{\bar{\alpha}}}\right)\right\rangle_{\Omega_{\kappa}, \Omega} \quad \text { for } \iota \in \mathfrak{J}_{\mathrm{P}},
$$

where the macroscale material derivative is defined as

$$
\frac{\mathrm{D}^{\overline{\bar{\alpha}}}}{\mathrm{D} t}=\frac{\partial}{\partial t}+\mathbf{W}^{\overline{\bar{\alpha}}} \cdot \nabla
$$

Eqn (50) states that, when following a volume fraction of a phase at a velocity $\mathbf{W}^{\overline{\bar{\alpha}}}$, the rate of change of that volume fraction is equal to the outward microscale velocity of the boundary of the phase, relative to $\mathbf{W}^{\bar{\alpha}}$, integrated over that boundary.

For a general three-phase system composed of two fluids and a solid, each phase has a boundary with the other two phases. Therefore, the boundary in eqn (50) is composed of two interfaces. For example, the $s$ phase is bounded by $n s$ and $w s$ interfaces. For subsequent derivation, it will be convenient to approximate the change in the volume of a phase in terms of the movement of only one of its boundary portions. When the solid is immobile, this can be achieved for the fluid phases without any approximation. For the general case of a slightly moving and deforming solid, we will assume that the normal velocity of each portion of the solid-phase boundary can be decoupled such that eqns (26) and (27) can be approximated, respectively, as

$$
\chi_{\kappa s}^{s s} \nabla \varepsilon^{s} \approx-\left\langle\mathbf{n}_{s}\right\rangle_{\Omega_{k s}, \Omega} \text { for } \kappa \in\{w, n\},
$$

and

$$
\chi_{\kappa s}^{s s} \frac{\partial \varepsilon^{s}}{\partial t} \approx\left\langle\mathbf{n}_{s} \cdot \mathbf{w}_{\kappa s}\right\rangle_{\Omega_{\kappa s}, \Omega} \quad \text { for } \kappa \in\{w, n\}
$$

where $\chi_{\kappa s}^{s s}$ is the fraction of the $s$ phase surface in contact with the fluid phase phase $\kappa$. With these approximations, the material derivative form of eqn (50) becomes

$$
\chi_{\kappa s}^{s s} \frac{\mathrm{D}^{\overline{\bar{\alpha}}} \varepsilon^{s}}{\mathrm{D} t} \approx\left\langle\mathbf{n}_{s} \cdot\left(\mathbf{w}_{\kappa s}-\mathbf{W}^{\overline{\bar{\alpha}}}\right)\right\rangle_{\Omega_{\kappa s}, \Omega} \quad \text { for } \kappa \in\{w, n\} .
$$


This approximation is exact when the deformation and movement of the solid is not dependent on which fluid it contacts. Summation of eqn (54) over all elements of the solid surface provides recovery of eqn (50) applied to the total solid-phase surface.

With incorporation of eqn (54), eqn (50) for a fluid phase may be approximated as

$$
\frac{\mathrm{D}^{\overline{\bar{\alpha}}} \varepsilon^{K}}{\mathrm{D} t}+\chi_{\kappa s}^{s s} \frac{\mathrm{D}^{\overline{\bar{\alpha}}} \varepsilon^{s}}{\mathrm{D} t} \approx\left\langle\mathbf{n}_{\kappa} \cdot\left(\mathbf{w}_{w n}-\mathbf{W}^{\overline{\bar{\alpha}}}\right)\right\rangle_{\Omega_{w n}, \Omega} \quad \text { for } \kappa \in\{w, n\} .
$$

This may also be expressed

$\varepsilon \frac{\mathrm{D}^{\overline{\bar{\alpha}}} s^{K}}{\mathrm{D} t}+\left(s^{\kappa}-\chi_{\kappa s}^{s s} \frac{\mathrm{D}^{\overline{\bar{\alpha}}} \varepsilon}{\mathrm{D} t} \approx\left\langle\mathbf{n}_{\kappa} \cdot\left(\mathbf{w}_{w n}-\mathbf{W}^{\overline{\bar{\alpha}}}\right)\right\rangle_{\Omega_{w n}, \Omega} \quad\right.$ for $\kappa \in\{w, n\}$,

where $\varepsilon$ is porosity and $s^{\kappa}$ is the saturation of fluid phase $\kappa$. Note that when the fluid saturation is equal to the fraction of the area of solid in contact with that fluid phase, the second term on the left hand side of eqn (56) will be zero. Additionally, if the solid phase movement alters the porosity with a much larger time constant than the rate at which saturation changes, the term involving the derivative of the porosity may be considered negligibly small.

The roles of eqns (54) and (55) or eqn (56) are to provide relations involving the averages over the surfaces that can be used in the equations for evolution of interfacial area. We can take the dot product of eqn (31) with $\mathbf{W}^{\overline{\bar{\alpha}}}$ and add the result to eqn (32) to obtain

$\frac{\mathrm{D}^{\overline{\bar{\alpha}}} \varepsilon^{l}}{\mathrm{D} t}+\nabla \cdot\left\langle\mathbf{n}_{\kappa} \mathbf{n}_{\kappa} \cdot\left(\mathbf{w}_{\iota}-\mathbf{W}^{\overline{\bar{\alpha}}}\right)\right\rangle_{\Omega_{l}, \Omega}+\nabla \cdot\left\langle\mathbf{n}_{\kappa} \mathbf{n}_{\kappa}\right\rangle_{\Omega_{l}, \Omega}: \delta^{\overline{\bar{\alpha}}}-\left\langle\left(\nabla^{\prime} \cdot \mathbf{n}_{\kappa}\right) \mathbf{n}_{\kappa} \cdot\left(\mathbf{w}_{\iota}-\mathbf{W}^{\overline{\bar{\alpha}}}\right)\right\rangle_{\Omega_{l}, \Omega}-\left\langle\mathbf{n}_{\iota} \cdot\left(\mathbf{w}_{w n s}-\mathbf{W}^{\overline{\bar{\alpha}}}\right)\right\rangle_{\Omega_{w n s}, \Omega}=0 \quad$ for $\iota \in \mathfrak{J}_{\mathrm{I}}$,

where the rate of strain tensor for the $\mathbf{W}^{\overline{\bar{\alpha}}}$ velocity is

$$
\left.\delta^{\overline{\bar{\alpha}}}=\frac{1}{2}\left\lceil\nabla \mathbf{W}^{\overline{\bar{\alpha}}+\left(\nabla \mathbf{W}^{\overline{\bar{\alpha}}}\right)}\right)^{T}\right\rceil .
$$

Next we define the average macroscale surface velocity normal to the interface, $\mathbf{w}^{\overline{\bar{\tau}}}$ as

$$
\mathbf{w}^{\bar{i}}=\left\langle\mathbf{n}_{\kappa} \mathbf{n}_{K} \cdot \mathbf{w}_{\iota}\right\rangle_{\Omega_{l}, \Omega_{l}} .
$$

Note that this definition provides an average of the normal component of the surface velocity. Then if we make use of the fact that the macroscale velocity $\mathbf{W}^{\overline{\bar{\alpha}}}$ can be removed from the averaging operator, we can introduce the definitions of the orientation tensors and the curvature given in eqns (33) and (34) and rearrange the terms to obtain 


$$
\begin{aligned}
\frac{\mathrm{D}^{\overline{\bar{\alpha}}} \varepsilon^{\iota}}{\mathrm{D} t} & \\
& +\nabla \cdot\left[\varepsilon^{\iota}\left(\mathbf{w}^{\overline{\bar{l}}}-\mathbf{G}^{\iota} \cdot \mathbf{W}^{\overline{\bar{\alpha}}}\right)\right] \\
& +\varepsilon^{\iota} \mathbf{G}^{\iota}: \delta^{\overline{\bar{\alpha}}} \\
& -J_{\kappa}^{\iota}\left\langle\mathbf{n}_{\kappa} \cdot\left(\mathbf{w}_{\iota}-\mathbf{W}^{\overline{\bar{\alpha}}}\right)\right\rangle_{\Omega_{\iota}, \Omega} \\
& -\left\langle\left(\nabla^{\prime} \cdot \mathbf{n}_{\kappa}-J_{\kappa}^{\iota}\right) \mathbf{n}_{\kappa} \cdot\left(\mathbf{w}_{\iota}-\mathbf{W}^{\overline{\bar{\alpha}}}\right)\right\rangle_{\Omega_{l}, \Omega} \\
& -\left\langle\mathbf{n}_{\iota} \cdot\left(\mathbf{w}_{w n s}-\mathbf{W}^{\overline{\bar{\alpha}}}\right)\right\rangle_{\Omega_{w n s}, \Omega} \\
& =0 \quad \text { for } \iota \in \mathfrak{J}_{I}, \\
& \text { and } \kappa \in\left(\mathfrak{J}_{a} \cap \mathfrak{J}_{\mathrm{P}}\right) .
\end{aligned}
$$

Although eqn (60) applies to the three different interface types, the behavior of the solidfluid interface is different from that of the fluid-fluid interface, so we will consider these two classes of interfaces separately in applying approximations geared to allow the remaining three terms involving averaging operators to be evaluated.

For the solid-fluid interface, we will make use of approximate eqn (54) so that eqn (60) becomes

$$
\begin{aligned}
\frac{\mathrm{D}^{\overline{\bar{\alpha}}} \varepsilon^{\iota}}{\mathrm{D} t} & \\
& +\nabla \cdot\left[\varepsilon^{l}\left(\mathbf{w}^{\overline{\bar{t}}}-\mathbf{G}^{\iota} \cdot \mathbf{W}^{\overline{\bar{\alpha}}}\right)\right] \\
& +\varepsilon^{\iota} \mathbf{G}^{\iota}: \delta^{\overline{\bar{\alpha}}} \\
& -J_{s}^{\iota} \chi_{\iota}^{s s} \frac{\mathrm{D}^{\overline{\bar{\alpha}}} \varepsilon^{s}}{\mathrm{D} t} \\
& -\left\langle\left(\nabla^{\prime} \cdot \mathbf{n}_{s}-J_{s}^{\iota}\right) \mathbf{n}_{s} \cdot\left(\mathbf{w}_{\iota}-\mathbf{W}^{\overline{\bar{\alpha}}}\right)\right\rangle_{\Omega_{\iota}, \Omega} \\
& -\left\langle\mathbf{n}_{\iota} \cdot\left(\mathbf{w}_{w n s}-\mathbf{W}^{\overline{\bar{\alpha}}}\right)\right\rangle_{\Omega_{w n s}, \Omega} \approx 0 \quad \text { for } \iota \in\{w s, n s\} .
\end{aligned}
$$

We will assume that the velocities of material points on the solid surface do not depend on which fluid phase they contact. We also assume that the orientation tensors for the solid surface are independent of the fluid phase. These conditions provide the useful relations

$$
\begin{gathered}
\mathbf{v}_{s}^{w s} \approx \mathbf{v}_{s}^{n s} \approx \mathbf{v}_{s}^{s s} \approx\left\langle\mathbf{v}_{s}\right\rangle_{\Omega_{\kappa s}, \Omega_{\kappa s}} \text { for } \kappa \in \mathfrak{J}_{\mathrm{p}} \\
\mathbf{G}^{w s} \approx \mathbf{G}^{n s} \approx \mathbf{G}^{s s} .
\end{gathered}
$$

For both the $w s$ and $n s$ interfaces, we select $\mathbf{W}^{\overline{\bar{\alpha}}}=\mathbf{v}_{s}^{s s}$ so that the material derivative is moving with the average velocity of the material points on the solid surface boundary. As an additional assumption, we assume that the integral involving the product of the curvature deviation and the normal velocity deviation is negligibly small for the solid surface such that 


$$
\left\langle\left(\nabla^{\prime} \cdot \mathbf{n}_{s}-J_{s}^{\iota}\right) \mathbf{n}_{s} \cdot\left(\mathbf{w}_{\iota}-\mathbf{v}_{s}^{s s}\right)\right\rangle_{\Omega_{l} \Omega} \approx 0 \text { for } \iota \in\{w s, n s\}
$$

With these conditions, eqn (61) can be written as

$$
\frac{\mathrm{D}^{s s} \varepsilon^{\iota}}{\mathrm{D} t}+\nabla \cdot\left[\varepsilon^{\iota}\left(\mathbf{w}^{\bar{i}}-\mathbf{G}^{s s} \cdot \mathbf{v}_{s}^{s s}\right)\right]+\varepsilon^{\iota} \mathbf{G}^{s s}: \delta^{\overline{\bar{s}}}-J_{s}^{\iota} \chi_{\iota}^{s s} \frac{\mathrm{D}^{s s} \varepsilon^{s}}{\mathrm{D} t}-\left\langle\mathbf{n}_{\iota} \cdot\left(\mathbf{w}_{w n s}-\mathbf{v}_{s}^{s s}\right)\right\rangle_{\Omega_{w n s}, \Omega} \approx 0 \quad \text { for } \iota \in\{w s, n s\}
$$

where

$$
\frac{\mathrm{D}^{s s}}{\mathrm{D} t}=\frac{\partial}{\partial t}+\mathbf{v}_{s}^{s s} \cdot \nabla
$$

and

$$
\delta^{\overline{\bar{s}}}=\frac{1}{2}\left[\nabla \mathbf{v}_{s}^{s s}+\left(\mathbf{v}_{s}^{s s}\right)^{\mathrm{T}}\right]
$$

In consideration of the definition given by eqn (59) and the assumption that the orientation tensors for the solid-fluid interfaces are equal, a reasonable approximation is

$\mathbf{w}^{\overline{\bar{i}}}=\left\langle\mathbf{n}_{\kappa} \mathbf{n}_{\kappa} \cdot \mathbf{w}_{\iota}\right\rangle_{\Omega_{t}, \Omega_{t}} \approx \mathbf{G}^{s s} \cdot\left\langle\mathbf{v}_{s}\right\rangle_{\Omega_{s s}, \Omega_{s s}}=\mathbf{G}^{s s} \cdot \mathbf{v}_{s}^{s s}$ for $\iota \in\{w s, n s\}$,

where we are neglecting mass exchange between the solid and fluid that causes a difference between the material normal velocity and the surface normal velocity to be significant. This approximation involves the subtle idea that the average of the normal velocity of the surface may be approximated as the orientation tensor on the surface dotted with the average of the velocity of material points on the surface. Material points may have velocities tangent to the surface. The orientation tensor has the effect of diminishing, if not eliminating, that contribution to an approximate macroscale surface velocity. Approximate eqn (68) has the effect of eliminating the second term in eqn (65) so that the equation for a fluid-solid interface reduces to

$$
\frac{\mathrm{D}^{s s} \varepsilon^{\iota}}{\mathrm{D} t}+\varepsilon^{\iota} \mathbf{G}^{s s}: \delta^{\overline{\overline{s s}}}-J_{s}^{\iota} \chi_{\iota}^{s s} \frac{\mathrm{D}^{s s} \varepsilon^{s}}{\mathrm{D} t}-\left\langle\mathbf{n}_{\iota} \cdot\left(\mathrm{w}_{w n s}-\mathrm{v}_{s}^{s s}\right)\right\rangle_{\Omega_{w n s}, \Omega} \approx 0 \quad \text { for } \iota \in\{w s, n s\}
$$

The last term on the left side of this equation accounts for an increase in the area density of a fluid-solid interface due to wetting of the solid. If we introduce the fact that $\varepsilon^{\iota}=\chi_{\iota}^{s s} \varepsilon^{s s}$ into this equation, we obtain

$$
\chi_{\iota}^{s s} \frac{\mathrm{D}^{s s} \varepsilon^{s s}}{\mathrm{D} t}+\varepsilon^{s s} \frac{\mathrm{D}^{s s} \chi_{\iota}^{s s}}{\mathrm{D} t}+\chi_{\iota}^{s s} \varepsilon^{s s} \mathbf{G}^{s s}: \delta^{\overline{\overline{s s}}}-J_{s}^{\iota} \chi_{\iota}^{s s} \frac{\mathrm{D}^{s s} \varepsilon^{s}}{\mathrm{D} t}-\left\langle\mathbf{n}_{\iota} \cdot\left(\mathbf{w}_{w n s}-\mathbf{v}_{s}^{s s}\right)\right\rangle_{\Omega_{w n s}, \Omega} \approx 0 \quad \text { for } \iota \in\{w s, n s\} .
$$

Summation of eqn (69) over the $w s$ and $n s$ interfaces causes the material derivative of the wetted solid fraction term to cancel out, and we obtain 


$$
\frac{\mathrm{D}^{s s} \varepsilon^{s s}}{\mathrm{D} t}+\varepsilon^{s s} \mathbf{G}^{s s}: \delta^{\overline{\overline{s s}}}-J_{s}^{s s} \frac{\mathrm{D}^{s s} \varepsilon^{s}}{\mathrm{D} t} \approx 0 .
$$

A representation of the wetting term that allows both eqns (70) and (71) to hold is

$$
\varepsilon^{s s} \frac{\mathrm{D}^{s s} \chi_{t}^{s s}}{\mathrm{D} t}=\left\langle\mathbf{n}_{l} \cdot\left(\mathbf{w}_{w n s}-\mathbf{v}_{s}^{s s}\right)\right\rangle_{\Omega_{w n s}, \Omega} .
$$

This term accounts for the change in interfacial area due to the wetting process alone, while an increase in area due to mechanical deformations is accounted for by other terms. Substitution of eqn (72) into eqn (69) gives the approximate forms for the fluid-solid interfacial areas

$$
\frac{\mathrm{D}^{s s} \varepsilon^{w s}}{\mathrm{D} t}+\varepsilon^{w s} \mathbf{G}^{w s}: \delta^{\overline{\overline{s s}}}-J_{s}^{w s} \chi_{w s}^{s s} \frac{\mathrm{D}^{s s} \varepsilon^{s}}{\mathrm{D} t}-\left(\varepsilon^{w s}+\varepsilon^{n s}\right) \frac{\mathrm{D}^{s s} \chi_{w s}^{s s}}{\mathrm{D} t} \approx 0
$$

and

$$
\frac{\mathrm{D}^{s s} \varepsilon^{n s}}{\mathrm{D} t}+\varepsilon^{n s} \mathbf{G}^{n s}: \delta^{\overline{\overline{s s}}}-J_{s}^{n s} \chi_{n s}^{s s} \frac{\mathrm{D}^{s s} \varepsilon^{s}}{\mathrm{D} t}-\left(\varepsilon^{w s}+\varepsilon^{n s}\right) \frac{\mathrm{D}^{s s} \chi_{n s}^{s s}}{\mathrm{D} t} \approx 0 .
$$

We will consider wetting to be a dynamic process that can be modeled according to

$$
\frac{\mathrm{D}^{s s} \chi_{w s}^{s s}}{\mathrm{D} t} \approx-\widehat{k}^{w s}\left(\chi_{w s}^{s s}-\chi_{w s e q}^{s s}\right)
$$

where the equilibrium wetted fraction as a function of saturation and average curvature of the fluid-fluid interface is

$$
\chi_{w s e q}^{s s}=\chi_{w s e q}^{s s}\left(s^{w}, J_{w}^{w n}\right),
$$

and $\hat{k}^{w s}$ is wetting rate parameter.

Now we return to eqn (60) for the fluid-fluid interface, denoted as the $w n$ interface. We can make use of the identity given as eqn (36) to express $\mathbf{n}_{w n}$ in terms of $\mathbf{n}_{s}$ and $\mathbf{n}_{w s}$ in the integral over the common curve and obtain 


$$
\begin{aligned}
\frac{\mathrm{D}^{\overline{\bar{\alpha}}} \varepsilon^{w n}}{\mathrm{D} t} & \\
& +\nabla \cdot\left[\varepsilon^{w n}\left(\mathbf{w}^{\overline{\overline{w n}}}-\mathbf{G}^{w n} \cdot \mathbf{W}^{\overline{\bar{\alpha}}}\right)\right] \\
& +\varepsilon^{w n} \mathbf{G}^{w n}: \delta^{\overline{\bar{\alpha}}} \\
& -J_{w}^{w n}\left\langle\mathbf{n}_{w} \cdot\left(\mathbf{w}_{w n}-\mathbf{W}^{\overline{\bar{\alpha}}}\right)\right\rangle_{\Omega_{w n}, \Omega} \\
& -\left\langle\left(\nabla^{\prime} \cdot \mathbf{n}_{w}-J_{w}^{w n}\right) \mathbf{n}_{w} \cdot\left(\mathbf{w}_{w n}-\mathbf{W}^{\overline{\bar{\alpha}}}\right)\right\rangle_{\Omega_{w n}, \Omega} \\
& -\left\langle\mathbf{n}_{w s} \cdot\left(\mathbf{w}_{w n s}-\mathbf{W}^{\overline{\bar{\alpha}}}\right) \cos \varphi_{w s, w n}\right\rangle_{\Omega_{w n s}, \Omega} \\
& +\left\langle\mathbf{n}_{s} \cdot\left(\mathbf{w}_{w n s}-\mathbf{W}^{\overline{\bar{\alpha}}}\right) \sin \varphi_{w s, w n}\right\rangle_{\Omega_{w n s}, \Omega}=0 .
\end{aligned}
$$

We will employ the approximation that the contact angle terms can be decoupled from the velocity of the common curve. This assumption is probably better for the component of $\mathbf{w}_{w n s}$ in the direction normal to the surface (due to solid surface movement) than for the component of $\mathbf{w}_{w n s}$ in the direction tangent to the surface. Thus in making the assumption that $\cos \varphi_{w s, w n}$ can be decoupled, we are neglecting the contributions of the dynamic nature of the contact angle to the change in the $w n$ interfacial area density. With this approximation, averages of the cos and sin function can be removed from the integrand in eqn (77). Here, as with the fluid-solid interface, we select $\mathbf{W}^{\overline{\bar{\alpha}}}=\mathbf{v}_{s}^{s s}$ so that the material derivative is with respect to a macroscale measure of the solid-phase velocity. Eqn (77) becomes

$$
\begin{aligned}
\frac{\mathrm{D}^{s s} \varepsilon^{w n}}{\mathrm{D} t} & \\
& +\nabla \cdot\left[\varepsilon^{w n}\left(\mathbf{w}^{\overline{\overline{w n}}}-\mathbf{G}^{w n} \cdot \mathbf{v}_{s}^{s s}\right)\right] \\
& +\varepsilon^{w n} \mathbf{G}^{w n}: \delta^{\overline{\overline{s s}}} \\
& -J_{w}^{w n}\left\langle\mathbf{n}_{w} \cdot\left(\mathbf{w}_{w n}-\mathbf{v}_{s}^{s s}\right)\right\rangle_{\Omega_{w n}, \Omega} \\
& -\left\langle\left(\nabla^{\prime} \cdot \mathbf{n}_{w}-J_{w}^{w n}\right) \mathbf{n}_{w} \cdot\left(\mathbf{w}_{w n}-\mathbf{v}_{s}^{s s}\right)\right\rangle_{\Omega_{w n}, \Omega} \\
& -\left\langle\mathbf{n}_{w s} \cdot\left(\mathbf{w}_{w n s}-\mathbf{v}_{s}^{s s}\right)\right\rangle_{\Omega_{w n s}, \Omega} \cos \varphi^{\overline{\overline{w s w w n}}} \\
& +\left\langle\mathbf{n}_{s} \cdot\left(\mathbf{w}_{w n s}-\mathbf{v}_{s}^{s s}\right)\right\rangle_{\Omega_{w n s}, \Omega} \sin \varphi^{\overline{\overline{w s, w n}}} \approx 0,
\end{aligned}
$$

where the macroscale cosine and sine functions are defined in eqns (37) and (38), respectively.

The last two averaged expressions in eqn (78) that multiply trigonometric functions can be expressed in terms of macroscale quantities. Eqn (72) may be applied to the integral multiplying the cos function to obtain

$$
\left\langle\mathbf{n}_{w s} \cdot\left(\mathbf{w}_{w n s}-\mathbf{v}_{s}^{s s}\right)\right\rangle_{\Omega_{w n s}, \Omega} \cos \varphi^{\overline{\overline{w s, w n}}}=\varepsilon^{s s} \frac{\mathrm{D}^{s s} \chi_{t}^{s s}}{\mathrm{D} t} \cos \varphi^{\overline{\overline{w s, w n}}}
$$

For the multiplier of the sin function, we make use of an approximation that the integral of the normal velocity component of the common curve over the common curve can be 
approximated using the ratio of the common curve density to the solid surface area density as [30]

$$
\left\langle\mathbf{n}_{s} \cdot\left(\mathbf{w}_{w n s}-\mathbf{v}_{s}^{s s}\right)\right\rangle_{\Omega_{w n s}, \Omega} \approx \frac{\varepsilon^{w n s}}{\varepsilon^{w s}+\varepsilon^{n s}}\left\langle\mathbf{n}_{s} \cdot\left(\mathbf{w}_{s}-\mathbf{v}_{s}^{s s}\right)\right\rangle_{\Omega_{s s}, \Omega}
$$

Then eqn (50) may be employed to evaluate the average on the right to obtain the approximation to be used

$$
\left\langle\mathrm{n}_{s} \cdot\left(\mathbf{w}_{w n s}-\mathbf{v}_{s}^{s s}\right)\right\rangle_{\Omega_{w n s}, \Omega} \sin \varphi^{\overline{\overline{w s, w n}}} \approx \frac{\varepsilon^{w n s}}{\varepsilon^{w s}+\varepsilon^{n s}} \frac{\mathrm{D}^{s s} \varepsilon^{s}}{\mathrm{D} t} \sin \varphi^{\overline{\overline{w s, w n}}}
$$

Eqn (55) may be employed to evaluate the average expression that is premultiplied by $J_{w}^{w n}$ such that

$$
J_{w}^{w n}\left\langle\mathbf{n}_{w} \cdot\left(\mathbf{w}_{w n}-\mathbf{v}_{s}^{s s}\right)\right\rangle_{\Omega_{w n}, \Omega} \approx J_{w}^{w n}\left(\frac{\mathrm{D}^{s s} \varepsilon^{w}}{\mathrm{D} t}+\chi_{w s}^{s s} \frac{\mathrm{D}^{s s} \varepsilon^{s}}{\mathrm{D} t}\right) .
$$

Substitution of eqns (79), (80), and (82) into eqn (78) gives

$$
\begin{aligned}
\frac{\mathrm{D}^{s s} \varepsilon^{w n}}{\mathrm{D} t} & \\
& +\nabla \cdot\left[\varepsilon^{w n}\left(\mathbf{w}^{\overline{\overline{w n}}}-\mathbf{G}^{w n} \cdot \mathbf{v}_{s}^{s s}\right)\right] \\
& +\varepsilon^{w n} \mathbf{G}^{w n}: \delta^{\overline{\overline{s s}}} \\
& -J_{w}^{w n}\left(\frac{\mathrm{D}^{s s} \varepsilon^{w}}{\mathrm{D} t}+\chi_{w s}^{s s} \frac{\mathrm{D}^{s s} \varepsilon^{s}}{\mathrm{D} t}\right) \\
& -\left\langle\left(\nabla^{\prime} \cdot \mathbf{n}_{w}-J_{w}^{w n}\right)\right\rangle \mathbf{n}_{w} \cdot\left(\mathbf{w}_{w n}-\mathbf{v}_{s}^{s s}\right)_{\Omega_{w n}, \Omega} \\
& -\varepsilon^{s s} \frac{\mathrm{D}^{s s} \chi_{w s}^{s s}}{\mathrm{D} t} \cos \varphi^{\overline{\overline{w s, w n}}} \\
& +\frac{\varepsilon^{w n s}}{\varepsilon^{w s}+\varepsilon^{n s}} \frac{\mathrm{D}^{s s} \varepsilon^{s}}{\mathrm{D} t} \sin \varphi^{\overline{\overline{w s, w n}}} \approx 0 .
\end{aligned}
$$

An expression in the equation for the solid-fluid interface evolution similar to the remaining averaging expression was set to zero in eqn (64) when treating the evolution of the solid phase surface. However, the fluid-fluid interface exhibits different behavior because the changes in this interfacial area are due to reconfigurations of the fluid phases that can significantly alter the amount of area at short time scales. For the solid-fluid interface, the main alterations in the amount of area at a short time scale involve wetting of the solid surface rather than reshaping. Subscale reconfiguration of the fluid-fluid interface, for example due to coalescence or trapping of a phase can be important. We therefore define

$$
e^{\overline{\overline{w n}}}=\left\langle\left(\nabla^{\prime} \cdot \mathbf{n}_{w}-J_{w}^{w n}\right) \mathbf{n}_{w} \cdot\left(\mathbf{w}_{w n}-\mathbf{v}_{s}^{s s}\right)\right\rangle_{\Omega_{w n}, \Omega}
$$

In previous studies that made some use of the averaging theorems for area evolution [30,39], a term like $e^{\overline{\overline{w n}}}$ was neglected under the assumption that "near equilibrium" it will be small. 
However, we will retain it here for further discussion. Substitution of eqn (84) into eqn (83) then gives

$$
\begin{aligned}
\frac{\mathrm{D}^{s s} \varepsilon^{w n}}{\mathrm{D} t} & \\
& +\nabla \cdot\left[\varepsilon^{w n}\left(\mathbf{w}^{\overline{\overline{w n}}}-\mathbf{G}^{w n} \cdot \mathbf{v}_{s}^{s s}\right)\right] \\
& +\varepsilon^{w n} \mathbf{G}^{w n}: \delta^{\overline{\overline{s s}}} \\
& -J_{w}^{w n}\left(\frac{\mathrm{D}^{s s} \varepsilon^{w}}{\mathrm{D} t}+\chi_{w s}^{s s} \frac{\mathrm{D}^{s s} \varepsilon^{s}}{\mathrm{D} t}\right) \\
& -e^{\overline{\overline{w n}}} \\
& -\varepsilon^{s s} \frac{\mathrm{D}^{s s} \chi_{w s}^{s s}}{\mathrm{D} t} \cos \varphi^{\overline{\overline{w s, w n}}} \\
& +\frac{\varepsilon^{w n s}}{\varepsilon^{w s}+\varepsilon^{n s}} \frac{\mathrm{D}^{s s} \varepsilon^{s}}{\mathrm{D} t} \sin \varphi^{\overline{\overline{w s, w n}}} \approx 0 .
\end{aligned}
$$

In this equation, the remaining challenge is to provide an estimate of $e^{\overline{\overline{w n}}}$. An appropriate form is to be selected based on data or heuristically in light of insights concerning system behavior. One helpful thought experiment is to consider a case where two isolated regions of one fluid phase come together and then redistribute. We can easily imagine a scenario in which this happens when there is no change in total phase volume for any phase, where the macroscale interface velocity is zero, and where no additional wetting occurs. Such a scenario describes a subscale change in the amount of area per volume due to microscale movement of the interface. Under the conditions described, the only terms that are non-zero in eqn (85) are the first and $e^{\overline{\overline{w n}}}$. In consideration of this scenario, it seems that $e^{\overline{\overline{w n}}}$ accounts for generation of interfacial area under conditions in which phase volumes can remain constant and macroscale advection need not occur. Thus $e^{\overline{\overline{w n}}}$ represents the combined processes of coalescence and division of phase resulting in the destruction or creation of interfacial area. This could be important in cases where separate regions of a phase are either combining due to mobilization or when portions of a phase are being trapped and disconnected from other portions of the phase. These mechanisms are well known to occur in multiphase porous medium systems, as entrapment and mobilization of the non-wetting phase occurs routinely in such systems [e.g. 35,45].

Although the form of $e^{\overline{\overline{w n}}}$ should be a subject of experimentation, we can hypothesize that one possible form to investigate is a linear relation

$$
e^{\overline{\overline{w n}}} \approx-\widehat{k}^{w n}\left(\varepsilon^{w n}-\varepsilon_{\mathrm{eq}}^{w n}\right)
$$

where $\varepsilon_{\mathrm{eq}}^{w n}=\varepsilon_{\mathrm{eq}}^{w n}\left(s^{w}, J_{w}^{w n}\right)$ is an equilibrium function and $k^{w n}$ is a rate coefficient that is characteristic of the speed at which the interfacial area reaches an equilibrium configuration after a coalescence or break-up event. Making use of this constitutive form, we obtain a general fluid-fluid interface evolution equation from eqn (85) as 


$$
\begin{aligned}
\frac{\mathrm{D}^{s s} \varepsilon^{w n}}{\mathrm{D} t} & \\
& +\nabla \cdot\left[\varepsilon^{w n}\left(\mathbf{w}^{\overline{\overline{w n}}}-\mathbf{G}^{w n} \cdot \mathbf{v}_{s}^{s s}\right)\right] \\
& +\varepsilon^{w n} \mathbf{G}^{w n}: \delta^{\overline{\overline{s s}}} \\
& -J_{w}^{w n}\left(\frac{\mathrm{D}^{s s} \varepsilon^{w}}{\mathrm{D} t}+\chi_{w s}^{s s} \frac{\mathrm{D}^{s s} \varepsilon^{s}}{\mathrm{D} t}\right) \\
& +\widehat{k}^{w n}\left(\varepsilon^{w n}-\varepsilon_{\mathrm{eq}}^{w n}\right) \\
& -\varepsilon^{s s} \frac{\mathrm{D}^{s s} \chi_{w s}^{s s}}{\mathrm{D} t} \cos \varphi^{\overline{\overline{w s, w n}}} \\
& +\frac{\varepsilon^{w n s}}{\varepsilon^{w s}+\varepsilon^{n s}} \frac{\mathrm{D}^{s s} \varepsilon^{s}}{\mathrm{D} t} \sin \varphi^{\overline{\overline{w s, w n}}} \approx 0 .
\end{aligned}
$$

A particular less general case of possible interest is when the solid phase is immobile or, at least, the solid phase dynamics are much slower than the dynamics of the fluid-fluid interface. For this case, if we also make use of eqn (75), eqn (87) simplifies to

$$
\frac{\partial \varepsilon^{w n}}{\partial t}+\nabla \cdot\left(\varepsilon^{w n} \mathbf{w}^{\overline{w n}}\right)-J_{w}^{w n} \varepsilon \frac{\partial s^{w}}{\partial t}+\widehat{k}^{w n}\left(\varepsilon^{w n}-\varepsilon_{\mathrm{eq}}^{w n}\right)+\varepsilon^{s s} \widehat{k}^{w s}\left(\chi_{w s}^{s s}-\chi_{w s e q}^{s s}\right) \cos \varphi^{\overline{\overline{w s, w n}}} \approx 0 .
$$

We emphasize the fact that $\mathbf{w}^{\overline{\overline{w n}}}$, which is defined by eqn (59), is the average of the normal velocity of the surface, not the average velocity in normal and tangential directions of any mass that might be associated with the surface.

In this section, we have derived the general macroscale equations for the kinematics of solid-fluid interfaces, eqns (73) and (74), subject to eqn (75), and for the fluid-fluid interface, eqn (87), subject to eqn (75). The resultant equations are statements of evolution of the interfacial area densities in response to interface velocities. These equations are distinct from and independent of mass conservation equations and considerations for the interfaces. As such, they do not depend on mass density, surface tension, temperature or any other thermodynamic properties of the interface. The relations are geometric approximations that describe how a surface will change when the regions it separates move, deform, coalesce, and break-up. It is important to understand that approximations are employed in evaluating the integrals that arise in obtaining equations for deformation of interfacial area densities. Thus opportunities to evaluate the accuracy of these approximations may arise in subsequent experiments and computations that could lead to improved relations.

\section{Common Curve Evolution}

The principles and considerations involved in derivation of the averaging theorems for common curves are not unlike those employed in the last section when dealing with the interface between phases. Here, we will develop an approximation for macroscale common curve kinematics for a three-phase system composed of two fluids, denoted as the $w$ and $n$ phases, and a solid phase, denoted as $s$. These three phases can meet at a common curve. Because there is no fourth phase, we will consider the case where no common points are present.

To obtain the evolution equation for the wns curve, we take the dot product of the common curve gradient averaging theorem, eqn (42), with the arbitrary macroscale velocity $\mathbf{W}^{\bar{\alpha}}$ and 
add this to the macroscale average transport theorem for a common curve, eqn (43), to obtain

$\frac{\mathrm{D}^{\overline{\bar{\alpha}}} \varepsilon^{w n s}}{\mathrm{D} t}+\nabla \cdot\left\langle\left(\mathbf{I}-\mathbf{n}_{w n s} \mathbf{n}_{w n s}\right) \cdot\left(\mathbf{w}_{w n s}-\mathbf{W}^{\overline{\bar{\alpha}}}\right)\right\rangle_{\Omega_{w n s}, \Omega}+\left\langle\left(\mathbf{I}-\mathbf{n}_{w n s} \mathbf{n}_{w n s}\right)\right\rangle_{\Omega_{w n s},}: \delta^{\overline{\bar{\alpha}}}+\left\langle\left(\mathbf{n}_{w n s} \cdot \nabla^{\prime \prime} \mathbf{n}_{w n s}\right) \cdot\left(\mathbf{w}_{w n s}-\mathbf{W}^{\overline{\bar{\alpha}}}\right)\right\rangle_{\Omega_{w n s}, \Omega}=0$

Here the average velocity of the common curve in the direction normal to the common curve is defined as

$$
\mathbf{w}^{\overline{\overline{w n s}}}=\left\langle\left(\mathbf{I}-\mathbf{n}_{w n s} \mathbf{n}_{w n s}\right) \cdot \mathbf{w}_{w n s}\right\rangle_{\Omega_{w n s}, \Omega_{w n s}} .
$$

Then if we introduce the common curve orientation tensor, $\mathbf{G}^{w n s}$, defined in eqn (44), and the geodesic and normal curvatures as developed in eqns (45)-(49), we can rewrite eqn (89), without approximation, as

$$
\begin{aligned}
\frac{\mathrm{D}^{\overline{\bar{\alpha}}} \varepsilon^{w n s}}{\mathrm{D} t} & \\
& +\nabla \cdot\left[\varepsilon^{w n s}\left(\mathbf{w}^{\overline{\overline{w n s}}}-\mathbf{G}^{w n s} \cdot \mathbf{W}^{\overline{\bar{\alpha}}}\right)\right] \\
& +\varepsilon^{w n s} \mathbf{G}^{w n s}: \delta^{\overline{\bar{\alpha}}} \\
& +\kappa_{G}^{w n s}\left\langle\mathbf{n}_{w s} \cdot\left(\mathbf{w}_{w n s}-\mathbf{W}^{\overline{\bar{\alpha}}}\right)\right\rangle_{\Omega_{w n s}, \Omega} \\
& \left.\left.+\kappa_{N}^{w n s}\left\langle\mathbf{n}_{s} \cdot\left(\mathbf{w}_{w n s}-\mathbf{W}^{\overline{\bar{\alpha}}}\right)\right\rangle_{\Omega_{w n s}, \Omega} \overline{\overline{\bar{\alpha}}}\right)\right\rangle_{\Omega_{w n s}, \Omega} \\
& +\left\langle( \kappa _ { G w n s } - \kappa _ { G } ^ { w n s } ) \mathbf { n } _ { w s } \cdot \left(\mathbf{w}_{w n s}-\mathbf{W}\right.\right. \\
& +\left\langle\left(\kappa_{\text {Nwns }}-\kappa_{N}^{w n s}\right) \mathbf{n}_{s} \cdot\left(\mathbf{w}_{w n s}-\mathbf{W}^{\overline{\bar{\alpha}}}\right)\right\rangle_{\Omega_{w n s}, \Omega}=0 .
\end{aligned}
$$

Now select $\mathbf{W}^{\overline{\bar{\alpha}}}=\mathbf{v}_{s}^{s s}$, which is the same choice that was made with the interfaces. Then employ the approximations noted previously as eqns (72) and (81) so that the first two of the averaged expressions in eqn (91) can be eliminated to obtain

$$
\begin{aligned}
\frac{\mathrm{D}^{s s} \varepsilon^{w n s}}{\mathrm{D} t} & \\
& +\nabla \cdot\left[\varepsilon^{w n s}\left(\mathbf{w}^{\overline{\overline{w n s}}}-\mathbf{G}^{w n s} \cdot \mathbf{v}_{s}^{s s}\right)\right] \\
& +\varepsilon^{w n s} \mathbf{G}^{w n s}: \delta^{\overline{s s}} \\
& +\kappa_{G}^{w n s} \varepsilon^{s s} \frac{\mathrm{D}^{s s} \chi_{w s}^{s s}}{\mathrm{D} t} \\
& +\kappa_{N}^{w n s} \frac{\varepsilon^{w n s}}{\varepsilon^{w s}+\varepsilon^{n s}} \frac{\mathrm{D}^{s s} \varepsilon^{s}}{\mathrm{D} t} \\
& +\left\langle\left(\kappa_{G w n s}-\kappa_{G}^{w n s}\right) \mathbf{n}_{w s} \cdot\left(\mathbf{w}_{w n s}-\mathbf{v}_{s}^{s s}\right)\right\rangle_{\Omega_{w n s}, \Omega} \\
& +\left\langle\left(\kappa_{N w n s}-\kappa_{N}^{w n s}\right) n_{s} \cdot\left(\mathbf{w}_{w n s}-\mathbf{v}_{s}^{s s}\right)\right\rangle_{\Omega_{w n s}, \Omega} \approx 0 .
\end{aligned}
$$


The last two terms account for a redistribution of the common curve due to processes not associated with wetting or transport of common curve into a location. The second of these terms, involving the deviation of the normal curvature from its average and the deviation of the normal velocity of the solid surface from its average, should be negligible as it accounts for stretching of an existing common curve on a non-uniformly expanding solid. The deviation term involving the geodesic curvature could contribute to common curve formation when some rearrangement, such as straightening of a curve, disappearance of the curve due to coalescence of phase regions, or formation of a curve due to trapping occur. All of these processes are approximately accounted for as a rate process, denoted $e^{\overline{\overline{w n s}}}$, such that

$$
e^{\overline{\overline{w n s}}}=-\left\langle\left(\kappa_{G w n s}-\kappa_{G}^{w n s}\right) \mathbf{n}_{w s} \cdot\left(\mathbf{w}_{w n s}-\mathbf{v}_{s}^{s s}\right)\right\rangle_{\Omega_{w n s}, \Omega}-\left\langle\left(\kappa_{N_{w n s}}-\kappa_{N}^{w n s}\right) \mathbf{n}_{s} \cdot\left(\mathbf{w}_{w n s}-\mathbf{v}_{s}^{s s}\right)\right\rangle_{\Omega_{w n s}, \Omega},
$$

and eqn (92) may be written

$$
\frac{\mathrm{D}^{s s} \varepsilon^{w n s}}{\mathrm{D} t}+\nabla \cdot\left[\varepsilon^{w n s}\left(\mathbf{w}^{\overline{\overline{w n s}}}-\mathbf{G}^{w n s} \cdot \mathbf{v}_{s}^{s s}\right)\right]+\varepsilon^{w n s} \mathbf{G}^{w n s}: \delta^{\overline{\overline{s s}}}+\kappa_{G}^{w n s} \varepsilon^{s s} \frac{\mathrm{D}^{s s} \chi_{w s}^{s s}}{\mathrm{D} t}+\kappa_{N}^{w n s} \frac{\varepsilon^{w n s}}{\varepsilon^{w s}+\varepsilon^{n s}} \frac{\mathrm{D}^{s s} \varepsilon^{s}}{\mathrm{D} t}-e^{\overline{\overline{w n s}}} \approx 0 .
$$

A linearized approximation to the generation term that accounts for the reshaping of a common curve may be proposed as

$$
e^{\overline{\overline{w n s}}} \approx-\widehat{k}^{w n s}\left(\varepsilon^{w n s}-\varepsilon_{\mathrm{eq}}^{w n s}\right)
$$

where $\varepsilon_{\mathrm{eq}}^{w n s}=\varepsilon_{\mathrm{eq}}^{w n s}\left(s^{w}, J_{w}^{w n}\right)$ is the equilibrium common curve length density as a function of saturation and curvature of the $w n$ interface, and $\hat{k}^{w n s}$ is a common curve generation rate coefficient. Substitution of the assumed form of eqn (95) into eqn (94) then provides the expression

$$
\frac{\mathrm{D}^{s s} \varepsilon^{w n s}}{\mathrm{D} t}+\nabla \cdot\left[\varepsilon^{w n s}\left(\mathbf{w}^{\overline{\overline{w n s}}}-\mathbf{G}^{w n s} \cdot \mathbf{v}_{s}^{s s}\right)\right]+\varepsilon^{w n s} \mathbf{G}^{w n s}: \delta^{\overline{s s}}+\kappa_{G}^{w n s} \varepsilon^{s s} \frac{\mathrm{D}^{s s} \chi_{w s}^{s s}}{\mathrm{D} t}+\kappa_{N}^{w n s} \frac{\varepsilon^{w n s}}{\varepsilon^{w s}+\varepsilon^{n s}} \frac{\mathrm{D}^{s s} \varepsilon^{s}}{\mathrm{D} t}+\widehat{k}^{w n s}\left(\varepsilon^{w n s}-\varepsilon_{\mathrm{eq}}^{w n s}\right) \approx 0
$$

A simplification of eqn (96) may be obtained under conditions where the solid phase dynamics are much slower than the interface movement. Under these circumstances, terms involving movement of material in the solid surface may be eliminated from eqn (94). We will also employ the rate expression given as eqn (75) to obtain

$$
\frac{\partial \varepsilon^{w n s}}{\partial t}+\nabla \cdot\left(\varepsilon^{w n s} \mathbf{w}^{\overline{w n s}}\right)-\kappa_{G}^{w n s} \varepsilon^{s s} \widehat{k}^{w s}\left(\chi_{w s}^{s s}-\chi_{w s e q}^{s s}\right)+\widehat{k}^{w n s}\left(\varepsilon^{w n s}-\varepsilon_{\mathrm{eq}}^{w n s}\right) \approx 0 .
$$

Eqn (97) has the elements of a conservation equation for the specific common curve length. The first term is a local rate of change, the second is a net outward flux, the third accounts for the change in common curve length due to wetting, and the fourth is a rate of change of common curve length due to coalescence and break-up. However, it is crucial to understanding this equation that the macroscale velocity denoted $w^{\overline{\overline{i n s}}}$ defined in eqn (90) involves only the velocity components of the common curve that are normal to the common curve. 
In this section, we have developed expressions for the evolution of common curve densities based on kinematic considerations and mathematical averaging theorems. This development confirms that the evolution of the internal geometry of the porous medium system does not depend on conservation equations for mass, momentum, or energy. The general approximation for the evolution of the common curve density is obtained as eqn (96) and was simplified for the case of negligible solid phase dynamics to eqn (97). The possibility of further subsequent improvement of the approximations employed here in light of experimental and computational evidence remains. Certainly, experimental support is needed to obtain the functional forms of the various rate coefficients and equilibrium distributions employed in the evolution equations.

One additional important challenge is obtaining a reasonable expression for the common curve velocity. Thus, we now turn to the task of obtaining useful estimates of the average of the normal surface velocities, defined in eqn (59). Note that approximations for the velocity of the solid surface were provided in eqn (68), but a corresponding form for the fluid-fluid interface has not been addressed. The macroscale common curve velocity defined in eqn (90) will also be examined to obtain a possible approximate macroscale form.

\section{Interface and Common Curve Velocities}

Equations that describe the macroscale evolution of interfacial areas and common curve length densities in response to averaged velocity distributions of these entities have been developed in the previous sections. It is very important to realize that the microscale velocities that contribute to changes in area or length densities are normal to the surfaces and the curve and are not related to the velocity of any material that may be transported within the surfaces or curve. For example, in a general case, the velocity distribution of any surface elements can be specified arbitrarily, and the deformations of the surface and its change in area can be studied based on that specified velocity field. The location of any surface that we might wish to study need not be at an interface between phases. It can be located arbitrarily at the microscale, and we can examine its extent, shape, and motion in response to an imposed velocity field. When we decide to study a surface or curve that happens to coincide with a boundary between phases or a location where three phases meet, we are imposing a constraint on the velocity field that will cause the surface to deform. To model such a deformation, we must relate what was an arbitrary velocity field for the surface or curve that instigates its evolution to some movement of fluids. This does not mean that the area and common curve evolution equations are no longer independent equations, it simply means that we wish to examine them in context with conservation equations. Just as independently derived mass and momentum equations may be used in concert to describe flow in a homogeneous system, detailed modeling of porous medium systems requires that we employ the independently derived equations of mass and momentum in concert with the evolution equations for the surfaces and common curves that are defined to reside along interior boundaries. If such a surface or curve contains mass, a mass conservation equation for that entity would be employed in addition to phase mass balance equations. The momentum of mass within a surface or curve would also be described by a momentum conservation equation for that entity. Again, this would be an equation in addition to an area evolution equation and does not supplant it. The issue to be addressed in this section is how the macroscale interface and common curve velocities should be specified in light of system physics so that the evolution of geometric densities is appropriately described.

The fact that the velocity of a region under study and the velocity of the material within the region are different applies to volumes, interfaces, and common curves. For this reason, one selects a control volume when studying a flow in space and defines the movement and deformation of that volume in such a way that the study of the flow is made convenient. 
Similarly one could select a control surface to study the behavior of material within a surface of interest. For fluid flow in a porous medium system, questions arises as to where the surfaces exist and how they deform. These questions must be answered if a system is to be fully modeled. For macroscale modeling, these questions are answered in an average sense, and the velocities that appear in the macroscale equations for interfacial area and common curve evolution are averages of the velocities that must be chosen to define the microscale regions. We recognize that, in general, these velocities of the regions are not known.

The surfaces and curves that we wish to follow are positions that describe the regions where two phases or three interfaces come together, respectively. The movement of these positions might then be describable in relation to the movement of the phases that are being separated.

The idea that the velocity of the surface needs to be described constitutively in terms of aspects of the system being modeled has been recognized previously, for example in [43]. In that work, the system of interest involved the movement of bubbles in a liquid phase; no solid phase was present. An area transport equation was derived for the bubble surface, and the resultant macroscale equation contained both the average velocity of the mass contained within the bubbles and the average velocity of the points on the bubble surface. For a onedimensional problem of interest, the constitutive approximation was invoked that the average of the material interface velocity was equal to the average of the velocity of the material within the bubble.

For two-fluid phase flow in a porous medium system, the situation is more complex. The velocity of the interface between phases will depend strongly on the microscale configuration and distribution of the phases within the porous medium. The solid phase is usually relatively immobile, and the difference between the macroscale velocity of the solid phase and the macroscale average velocity of material solid particles at the solid-fluid interface is likely small. Therefore, the constitutive approximation will be made that

$$
\mathbf{v}_{s}^{s s} \approx \mathbf{v}^{\bar{s}}
$$

This equation is in addition to eqn (62) in which the velocity of the material on the solid surface was approximated as being independent of the contact fluid. Thus, to be consistent with eqn (68), the average normal velocity of the solid surfaces is related to the material velocity as

$$
\mathbf{w}^{\overline{\bar{i}}}=\mathbf{G}^{s s} \cdot \mathbf{v}^{\bar{s}} \text { for } \iota \in\{w s, n s\}
$$

The specification of $\mathbf{w}^{\overline{\overline{v n}}}$ requires information about the velocity of the fluids and the orientation of the $w n$ interface. We know that when the flow is steady, the amount of interface per volume is constant. The velocity of the fluid at the fluid-fluid interface is not necessarily zero, although, for some configuration, the normal velocity of the interface could be zero. In general, however, a condition of steady state is that the net outward flux of interface or common curve will be zero. Therefore, at steady state for the fluid-fluid interface and for the common curve we have, regardless of the fluid-phase velocities,

$$
\nabla \cdot\left(\varepsilon^{w n} \mathbf{w}^{\overline{\overline{w n}}}\right)=0
$$


and

$$
\nabla \cdot\left(\varepsilon^{w n s} \mathbf{w}^{\overline{\overline{w n s}}}\right)=0
$$

Note that in eqn (85), or its simplified form eqn (88), the divergence of $\varepsilon^{w n} \mathbf{w}^{\overline{\overline{w n}}}$ appears. Changes in $w n$ area due to phase expansion or contraction, to coalescence and breakup, and to the movement of the common curve are accounted for by other terms. Consider the case of a water-saturated system in which a non-wetting fluid phase displaces the wetting phase. Close to the water-saturated limit as a non-wetting phase begins to invade the system, it seems reasonable that a net flux of surface area would accompany the invasion of the non-

wetting phase such that $\nabla \cdot\left(\varepsilon^{w n} \mathbf{w}^{\overline{\overline{w n}}}\right)$ would be important. As drainage progresses to intermediate wetting phase saturations, the changes in saturation would result from wetting phase displacement through progressively smaller pore bodies and changes in the curvature of wetting phase non-wetting phase interfaces in wedges of the pore space previously drained. Under these conditions the divergence term would also be of importance, but less dominantly so than at the highest saturation levels. Finally in the limit of small wettingphase saturations as drainage in nearly complete, few pore bodies remain to be drained and the primary change in interfacial area would result from curvature changes in the wetting phase. Under these conditions, the divergence term would be relatively unimportant compared to the higher wetting-phase saturation levels.

These considerations suggest that $\mathbf{w}^{\overline{\overline{w n}}}$ will depend on the macroscale velocities of the fluid phases as well as the distribution of the phases in the medium. In fact, when there is no transfer of material across the $w n$ interface, the microscale velocity of the surface, $\mathbf{w}_{w n} \cdot \mathbf{n}_{w} \mathbf{n}_{w}$, will equal the normal component of the fluid phase velocity at the interface. If we consider that mass transfer has a negligible effect on the normal movement of a fluid interface, the difference between the interface velocity and the normal component of the fluid velocity at the interface may still be negligible. On this basis, we can hypothesize that the exact expression when no phase change occurs may also be applied as an approximation when phase change is occurring such that

$$
\mathbf{w}^{\overline{\overline{w n}}} \approx\left\langle\mathbf{v}_{w} \cdot \mathbf{n}_{w} \mathbf{n}_{w}\right\rangle_{\Omega_{w n}, \Omega_{w n}} \approx\left\langle\mathbf{v}_{n} \cdot \mathbf{n}_{n} \mathbf{n}_{n}\right\rangle_{\Omega_{w n}, \mathbf{\Omega}_{w n}} .
$$

The average phase velocity over the $w n$ interface is not generally equal to the velocity over the phase. However, the fact that only the normal component of the material velocity at the interface is of importance suggests that the interface velocity may be expressed constitutively as

$$
\mathbf{W}^{\overline{\overline{w n}}} \approx \mathbf{G}^{w n} \cdot\left(\widehat{A}^{w} \mathbf{v}^{\bar{w}}+\widehat{A}^{n} \mathbf{v}^{\bar{n}}\right),
$$

where interfacial velocity coefficients $\hat{A}^{w}$ and $\hat{A}^{n}$ are, perhaps, functions of the ratio of dynamic viscosities of the fluids $\hat{\mu}_{w} / \hat{\mu}_{n}, s^{w}$, and $\varepsilon^{w n}$. Experiments are needed to help identify further the functional form of these constitutive coefficients.

A constitutive relation for the velocity of the common curve to be used in eqn (96), or its simplified version eqn (97), is challenging to obtain. At the solid surface, the phase velocities are equal to the velocity of the solid. Movement of the common curve is actually 
not flow, in the usual sense, but is a result of complex fluid motion in the vicinity of the common curve. However, we consider wetting of a surface to be a phenomenon that is associated with common curve movement. When imbibition of a wetting fluid into a porous medium domain filled with a non-wetting phase occurs, common curves form at the front and move along the surface. This wetting phenomenon is accounted for by the term in eqn (96) or eqn (97) involving the geodesic curvature. When the wetting-phase saturation is high, the solid can be completely wet by the $w$ phase so that no common curves remain at the end of the process. Common curve dynamics will likely be most important at low wetting-phase saturations when non-wetting fluid is trapped. Development of expressions for the common curve advection velocity, $\mathbf{w}^{\overline{\overline{\overline{n n s}}}}$ seems to be a higher order demand that will take significant experimental and computational work. Note that this quantity will be zero at steady state and represents a process other than wetting of the surface. Thus, it may be satisfactory, at least as a first effort, to assume that the term involving $\mathbf{w}^{\overline{\overline{w n s}}}$ contributes negligibly to common curve length evolution. Another approximation may be to try to express the macroscale common curve velocity in terms of the two fluid phase velocities in a direction tangent to the solid surface.

If we consider the velocity of the common curve normal to the solid to be small since the common curve lies on the solid surface, the definition of eqn (90) may be approximated when the solid is immobile as

$$
\mathbf{w}^{\overline{\overline{w n s}}} \approx\left\langle\mathbf{n}_{w s} \mathbf{n}_{w s} \cdot \mathbf{w}_{w n s}\right\rangle_{\Omega_{w n s}, \Omega_{w n s}} .
$$

Then in terms of the velocity of the fluid phases, the common curve velocity may be postulated constitutively as

$$
\mathbf{w}^{\overline{\overline{w n s}}} \approx\left(\mathbf{G}^{w n s}-\mathbf{G}^{s s}\right) \cdot\left(\widehat{B}^{w} \mathbf{v}^{\bar{w}}+\widehat{B}^{n} \mathbf{v}^{\bar{n}}\right)
$$

where common curve velocity coefficients $\hat{B}^{w}$ and $\hat{B^{n}}$ might be assumed to depend on $\hat{\mu}_{w} / \hat{\mu}_{n}$, $s^{w}$, and $\varepsilon^{w n}$. In any event, the approach required for analysis of the macroscale net flux of common curves is not as clear, and likely not as important, as the approach to accounting for the fluid-fluid interface flux.

\section{Comparison with Previous Models}

The derivation here has resulted in evolution equations for the $w s$ and $n s$ interfaces (eqns (73) and (74) subject to eqn (75)), a general evolution equation for the wn fluid-fluid interface (eqn (87) subject to eqn (75)), a simplified evolution equation for the $w n$ interface (eqn (88)), a general evolution equation for the common curve formed at the juxtaposition of two fluid phases and a solid phase (eqn (96) subject to eqn (75)), and a simplified common curve evolution equation (eqn (97)). Of these, the equation for a fluid-fluid interface has received the most attention previously [37, 38, 41, 43, 57-60], so that a comparison with the forms that have been proposed seems warranted.

The fluid fluid interface equation proposed by Kalaydjian [41] is given as eqn (2). This equation is, in fact, a simplified form of eqn (88) that would apply when there is no net advection of interfacial area into a region, the porosity is constant, the $w n$ interface generation due to coalescence and break-up is negligible, and wetting of the solid is not accompanied by stretching of the interface. These restrictions are strong, but are consistent with the objective of providing a simple illustrative example. As has been mentioned, one of 
the equations involving the interface velocity used in [41], eqn (4), is not consistent with even a simplified multiphase system.

The model of Kim [43] and Ishii and Kim [37] was developed in a context with no solid phase present. Their simplified equation is provided here as eqn (10). Despite the very different systems considered, eqn (88) can be reduced to that equation when ${ }_{\mathbf{w}} \overline{\overline{w n}} \cdot \nabla \varepsilon^{w n}$ is small compared to the other terms in the equation; the orientation of the interface is isotropic (as would be expected for a surface bounding a bubble or set of bubbles) such that

$\mathbf{w}^{\overline{\overline{w n}}} \approx \mathbf{v}^{w n} ; J_{w}^{w n}=-2 \varepsilon^{w n} /\left(3 \varepsilon^{n}\right)$ as is the case when the $n$ phase is composed of bubbles; and the coalescence and break-up terms are prescribed to have the same form as the equations developed in this work. The selection of the velocity of the bubble surface in this model is consistent with eqn (103) with $\hat{A}^{w}=0, \hat{A}^{n}=1$, and $\mathbf{G}^{w n}=\mathbf{I} / 3$.

If one compares the model of Gray et al. [30] as expressed in eqn (14) with eqn (87) derived here, the similarities are numerous, especially if the contributions of the solid and solid surface dynamics are negligible. However, there are several very important differences. Here, the velocities are surface averages rather than mass averages. This is the correct approach since the effort is at modeling a geometric shape regardless of the mass density distribution in the surface. Also, no effort is made in [30] to ensure that only the normal component of the surface velocity is employed. Finally, eqn (14) was developed while considering that the system dynamics were slow enough that "near equilibrium" conditions prevailed such that the coalescence and break-up mechanisms could be neglected. Despite these shortcomings, the equations for the fluid-fluid interface, the fluid-solid interfaces, and the common curve provided a useful initial effort to employ averaging theorems to describe the dynamics of geometric regions.

We can further explore the utility and validity of eqn (21) proposed by Niessner and Hassanizadeh [57-60] in light of eqn (88), which was developed relying solely on mathematical theorems without recourse to conservation equations. We will examine whether reasonable conditions exist under which eqn (21) might be a useful evolution equation by comparing it with eqn (88). Both equations begin with a time derivative of the $w n$ interfacial area density. The next terms are divergence terms, but they are inherently different. The divergence term of eqn (21) involves the velocity of the mass in the interface. This macroscale velocity is an average based on the stipulation that the interface must have uniform, non-zero, mass density and it contains elements of both the tangential velocity of the flow and the normal velocity of the interface. Thus the change in interface density is proposed as being dependent on the speed at which material moves tangentially within the interface as well as on the distortion of the interface due to its normal motion. As formulated in [57-60], $\mathbf{v}^{w n}$ also has the property that it will be zero only if the gradient in $\varepsilon^{w n}$ is zero, unless all dynamics are accounted for in a coefficient. The divergence term derived here in eqn (88) is correctly based on the macroscale value of the normal component of the interface velocity.

The expression in [57-60] has no terms comparable to the last two in eqn (88) that account for change in interfacial area due to coalescence, break-up, and wetting of the solid phase surface. Thus eqn (21) is unable to simulate growth of interfacial area that could take place with a zero average interface velocity and at constant saturation. The multiplier of the time derivative of $s^{w}$ in eqn (88) is the curvature of the $w n$ interface, which is negative when capillary forces exist at the interface between the wetting $w$ and non-wetting $n$ phases. This coefficient is dependent on the state of the system having a more negative value at low saturations and approaching 0 as the saturation approaches 1 . It is stated in [57] that the parameter $\hat{e}^{w n}$, which multiplies the time derivative of $s^{w}$ in their eqn (21) is positive for drainage and negative for imbibition. The fact remains that formulation of the area transport 
equation based on mass conservation principles, but ultimately relying on a hypothesis, has been shown to differ fundamentally from the other perspectives outlined here $[3,21,30,37,38,41,56]$ that assert that interfacial area deformations and transport are independent of physical conservation principles. Evolution equations for interfacial areas and for common curves are related to mathematical differential geometry issues rather than fundamental physical conservation laws.

\section{Closed Two-Fluid-Phase Flow Model}

The previously derived equations for specific interfacial area evolution and the approximations of the normal velocity of these entities can be used to produce a closed twofluid-phase flow model using the TCAT approach. Because these new equations enter the TCAT formulation as closure relations, the previously detailed two-fluid-phase flow model given by Jackson et al. [39] can be supplemented with the new equations derived in this work to produce a closed model. Jackson et al. [39] assumed that the two fluid phases were continuous, such as would be the case when a water saturated porous medium was drained by a non-wetting phase. The general case of two fluid phase flow was not addressed because of the complications associated with the coalescence and break-up of individual fluid regions, which affect specific interfacial areas and common curve lengths. Because the formulation detailed above includes these mechanisms, the two-fluid-phase flow model of Jackson et al. [39] can be extended to the more general case of any combination of fluid morphologies and topologies without resorting to a need to rederive the entropy inequality portion of the formulation.

Our objective here is not to provide a model of great complexity that models an extremely broad range of porous medium situations. Rather, we wish to provide an indication of how the geometric evolution equations can enter a formulation. More specifically, we will examine a system in which the solid phase does not deform and the common curve density is considered to be described by an equation of state. This approach allows us to highlight how the fluid-fluid interfacial area density evolution equation fits within a formulation. The simple model presented here does not exploit the full potential of TCAT and the evolution equations in modeling a complex system. The equations presented are a subset of those considered in [39].

Interest here is in an isothermal porous medium system composed of two immiscible fluids and a rigid solid where no chemical reactions are occurring and mass exchange between phases is negligible. Additionally, we will consider the interfaces between phases, as well as the common curve, to be massless.

The macroscale mass conservation equations for the fluid phases are

$$
\frac{\partial\left(\varepsilon^{l} \rho^{l}\right)}{\partial t}+\nabla \cdot\left(\varepsilon^{\imath} \rho^{\iota} \mathbf{v}^{\bar{\imath}}\right)=0 \quad \text { for } \iota \in\{w, n\}
$$

In [39] it was shown that the driving force for flow is the sum of chemical and gravitational potentials. In many instances, it may be possible to approximate this force being related to the pressure gradient and gravity. If that situation is employed here, the momentum equations for the fluids employed are of a Darcy type with cross-coupling such that

$$
\widehat{\mathbf{R}}_{w}^{\iota} \cdot \mathbf{v}^{\bar{w}}+\widehat{\mathbf{R}}_{n}^{\iota} \cdot \mathbf{v}^{\bar{n}}=-\nabla p^{\iota}+\rho^{\imath} \mathrm{g} \quad \text { for } \iota \in\{w, n\},
$$


where the resistance tensors $\widehat{\mathbf{R}}_{\kappa}^{\iota}$ are functions of saturation and the interfacial areas. Equations of state will also be employed that related the fluid densities to pressure for the isothermal case of the forms

$$
\rho^{\iota}=\rho^{l}\left(p^{l}\right) \quad \text { for } \iota \in\{w, n\} .
$$

Eqns (106)-(108) comprise a set of 10 scalar equations in the 13 variables $s^{w}, \rho^{w}, \rho^{n}, \mathbf{v}^{\bar{w}}, \mathbf{v}^{n}$, $p^{w}, p^{n}, \varepsilon^{w n}$, and $\varepsilon^{w s}$. Thus, we have a deficit of three equations. We note that in standard approaches to modeling this set of equations, the dependence of resistance on the interfacial areas is not included so that only 11 variables are involved, and the equation deficit is one. For such a case, the closure is achieved by proposing a capillary pressure function that relates the two pressures and saturations. A more general approach is employed here that includes the interfacial areas such that we are in need of three additional equations.

The capillary pressure relation will be considered that accounts for disequilibrium between the pressures on each side of the interface between phases and the curvature needed for equilibrium between the pressures to hold. In [39], it was shown that the correct formulation of the capillary pressure involves averages of phase pressures over the interface. Some additional work is needed to arrive at an improved formulation for the capillary pressure that accounts for the dynamics of the interface and the fact that the phase average pressure is typically employed. That work will be postponed for the time being and, instead, we employ the common expression

$$
\varepsilon \frac{\partial s^{w}}{\partial t}=\frac{1}{\widehat{c}^{w n}}\left(p^{w}-p^{n}-\gamma^{w n} J_{w}^{\overline{\overline{w n}}}\right) .
$$

In this form, the product $-\gamma^{w n} J_{w}^{\overline{\overline{w n}}}$ is typically identified as an equilibrium capillary pressure [55]. Here, the explicit presence of the interfacial curvature is interesting because it denotes a geometric situation. We will consider that the macroscale curvature may be expressed as a function of saturation and the interfacial area densities. Note that for a phase present in a sphere or collection of spheres, the curvature of the boundary of the interface will be uniquely defined if the phase volume and bounding area are known. For the porous medium case, we adopt a somewhat more general functional form and assume

$$
J_{w}^{\overline{\overline{w n}}}=J_{w}^{\overline{\overline{w n}}}\left(s^{w}, \varepsilon^{w n}, \varepsilon^{w s}\right) .
$$

Eqns (109) and (110) add two equations to the set, but only one variable, $J_{w}^{\overline{\overline{w n}}}$, such that the equation deficit is reduced to two. A constitutive relation will be needed for the additional parameter $\hat{c}^{w n}$.

The needed additional equations are obtained as evolution equations for the $w s$ and $w n$ interface. In this simple model, the common curve dynamics are considered unimportant so that, in the rate expression for wetting of the solid given by eqn (75), we consider $\hat{k}^{w s}$ to be very large so that

$$
\chi_{w s}^{s s}=\chi_{w s e q}^{s s}\left(s^{w}, \varepsilon^{w n}\right)
$$

Multiplication of this constitutive function by $\varepsilon^{s s}$ provides the expression for $\varepsilon^{w s}$ as 


$$
\varepsilon^{w s}=\varepsilon^{s s} \chi_{w \text { seq }}^{s s}\left(s^{w}, \varepsilon^{w n}\right)
$$

The final equation needed to close this system is obtained from eqn (87) which may be simplified for the case of an immobile, non-deforming solid to

$\frac{\partial \varepsilon^{w n}}{\partial t}+\nabla \cdot\left(\varepsilon^{w n} \mathbf{w}^{\overline{w n}}\right)-J_{w}^{w n} \varepsilon \frac{\partial s^{w}}{\partial t}+\widehat{k}^{w n}\left(\varepsilon^{w n}-\varepsilon_{\mathrm{eq}}^{w n}\right)-\frac{\partial \varepsilon^{w s}}{\partial t} \cos \varphi^{\overline{\overline{w s, w n}}} \approx 0$.

This equation requires support from a constitutive expression for $\mathbf{w}^{\overline{\overline{w n}}}$ as given by eqn (103) and repeated here for completeness

$$
\mathbf{w}^{\overline{\overline{w n}}}=\mathbf{G}^{w n} \cdot\left(\widehat{A}^{w} \mathbf{v}^{\bar{w}}+\widehat{A}^{n} \mathbf{v}^{\bar{n}}\right)
$$

Constitutive functional forms for the coefficients $\hat{A}^{w}\left(\varepsilon^{w n}, s^{w}\right), \hat{A}^{n}\left(\varepsilon^{w n}, s^{w}\right) \hat{k}^{w n}\left(\varepsilon^{w n} ; s^{w}\right)$, $\varepsilon_{\mathrm{eq}}^{w n}\left(\varepsilon^{w n}, s^{w}\right)$, and $\cos \varphi^{\overline{\overline{w s, w n}}}$ must also be supplied so that the equation system is fully closed. The geometric orientation tensor, $\mathbf{G}^{w n}$ is also required. As a first estimate, one might consider isotropic interface orientation such that $\mathbf{G}^{w n}=\mathbf{I} / 3$. In any event, the equation set reveals the potentially important parameters, each of which can be related to operative physical processes in the system, when the interfacial area density is to be included in a twofluid phase porous medium flow model.

\section{Discussion}

The purpose of this paper has been to establish relationships for the evolution of interfaces and the common curve in two-fluid-phase porous medium systems. Equations have been developed for interfaces between the solid and each of the fluid phases as well as for the interface between the two fluid phases. Additionally, an expression was obtained for the evolution of the common curve at the solid surface where the three phases meet. These equations were developed on the basis of mathematical statements that provide information about changes in the area of a surface or the length of a curve as a result of the motion of points on these entities. These equations are independent relations that do not depend on conservation of mass, momentum, or energy. This observation is consistent with that of approaches taken previously in continuum mechanics, bubbly flow modeling, and some porous medium analyses. The velocities that appear in the evolution equations are average entity velocities, not mass-weighted velocities. Also, constitutive equations for the macroscale interface and common curve velocities have been advanced.

Based on the equations developed, a relatively simple closed model for two phase flow has been proposed. This model provides a generalization of usual two-phase flow models in that the fluid-fluid interfacial area density is incorporated into the model. The formulation gives rise to a number of constitutive functions that must be developed. Although the overhead may seem daunting, the framework provided here is physically and mathematically consistent such that it lends itself to subsequent experimental and computational studies that should provide useful correlations and parameterizations. Of course, if one presumes that interfacial areas are not important, the generalized model here reduces to commonly used forms. 
Although the closed model provided here is for the case where the solid is immobile, and the common curve kinematics are not included, the full framework has been provided for including these effects.

\section{Notation}

\section{Roman letters}

$\hat{A} \quad$ constitutive coefficient for interface velocity

$\widehat{B} \quad$ constitutive coefficient for common curve velocity

d rate of strain tensor

da differential element of surface

$\hat{\mathbf{e}}^{w n} \quad$ interfacial area generation

$\boldsymbol{e} \quad$ generation term due to coalescence and break up

e unit outward normal endpoint vector for a common curve

$f \quad$ general function

$f_{\mathbf{l}} \quad$ general microscale property

G Geometric tensor

I identity tensor

$\Im$ index set of entities

$\Im_{\mathbf{c}} \quad$ index set of connected entities

$\Im_{\mathrm{C}} \quad$ index set of common curves

$\Im_{f} \quad$ index set of fluid-phase entities

$\mathfrak{I}_{\mathbf{I}} \quad$ index set of interfaces

$\mathfrak{I}_{\mathbf{P}} \quad$ index set of phase entities

Ji surface curvature defined as the surface divergence of the outward normal from entity 1

$J_{\kappa}^{\iota} \quad$ macroscale curvature of the $\mathrm{\imath}$ interface obtained as average of $\nabla^{\prime} \cdot \mathbf{n}_{\kappa}$ over the interface over the $\mathrm{i}$ interface

$\hat{\mathbf{K}}^{w n} \quad$ interfacial permeability tensor

$\hat{\boldsymbol{k}} \quad$ generation rate coefficient

$\boldsymbol{L}_{\mathbf{1}} \quad$ model parameter

$\boldsymbol{L}_{2} \quad$ model parameter

$\stackrel{\kappa \rightarrow}{M} \quad$ rate of transfer of mass per volume from the $\kappa$ to the tentity

$\mathbf{n}_{\mathbf{l}} \quad$ outward normal vector from entity $\mathbf{l}$ on its boundary when $\mathrm{\imath} \in \Im_{\mathrm{P}}$, tangent vector to surface and outward normal from the bounding common curve $\imath$ when $\imath \in \Im_{I}$, and tangent to the common curve when $\mathrm{i} \in \Im_{\mathrm{C}}$

microscale quantity

$\boldsymbol{p} \quad$ fluid pressure

$\boldsymbol{p}^{\boldsymbol{c}} \quad$ macroscale capillary pressure

R resistance tensor 
$\boldsymbol{r} \quad$ rate of generation

$s \quad$ saturation

$t \quad$ time

$\boldsymbol{V} \quad$ size of averaging volume

v velocity

w velocity of the interface or common curve

$\boldsymbol{W} \quad$ weighting function

W arbitrary reference macroscale velocity

$\mathbf{w}^{\prime} \quad$ velocity of the surface tangent to the surface

\section{Greek letters}

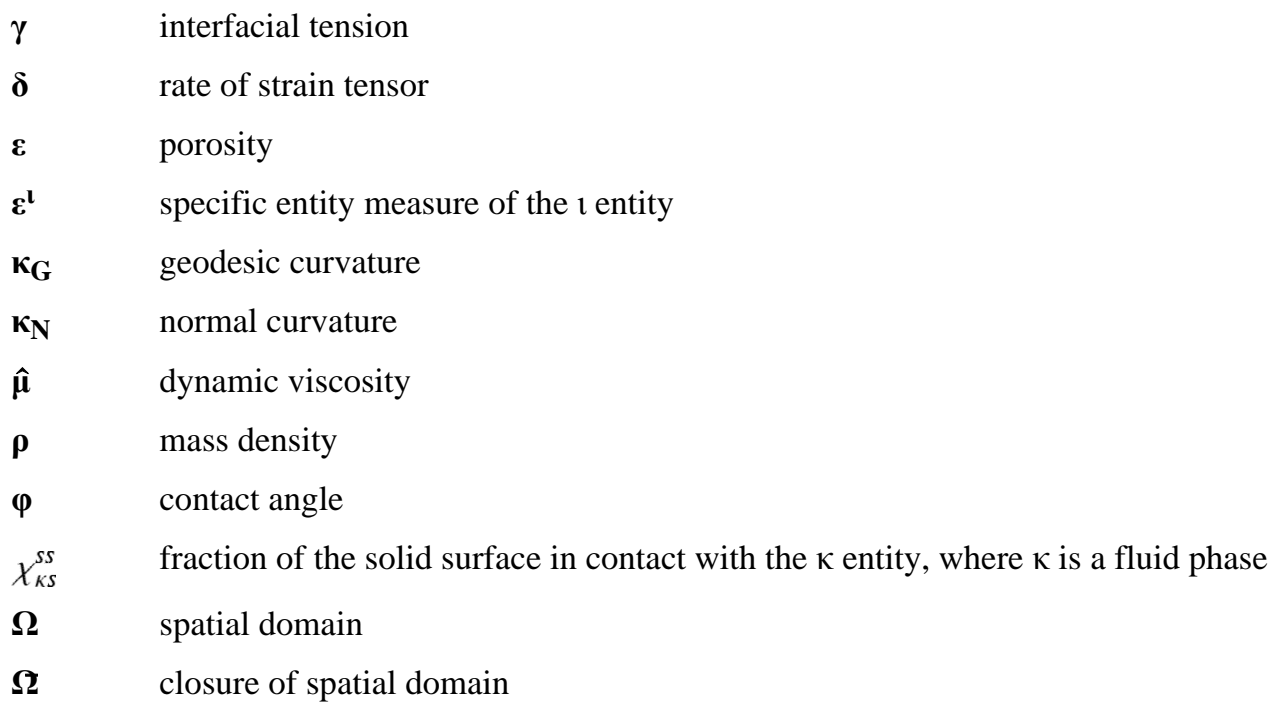

\section{Subscripts (for microscale) and superscripts (for macroscale)}

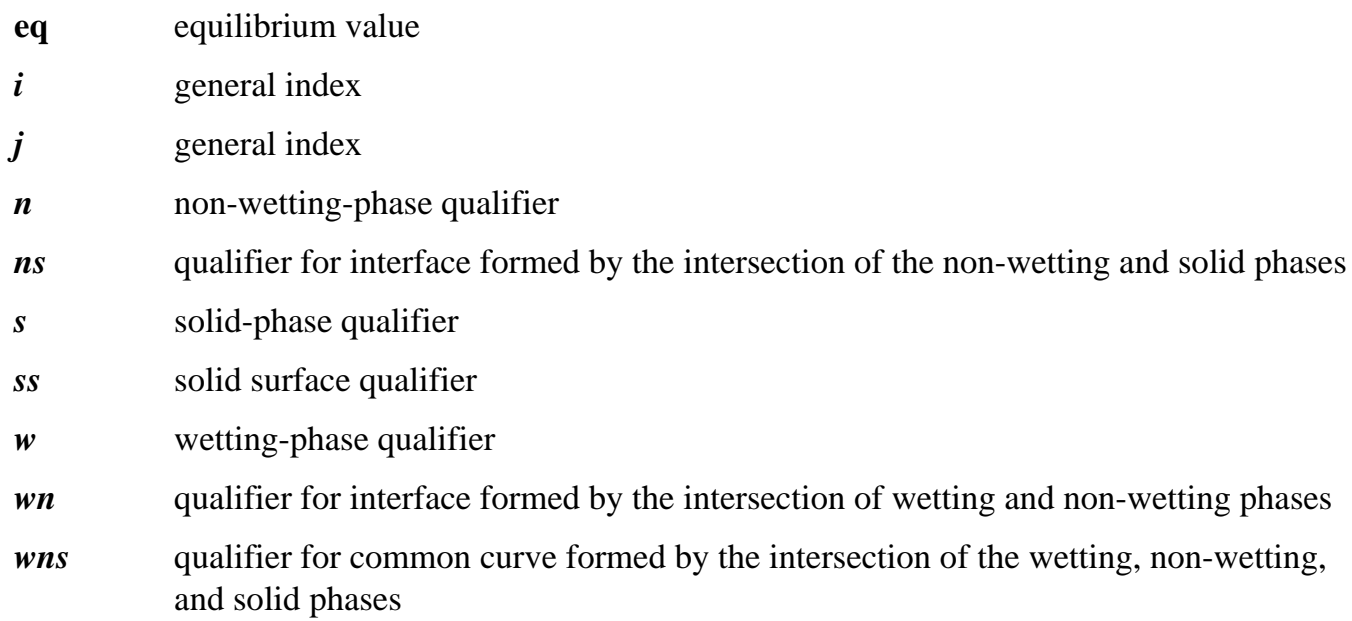


$w s$

$\boldsymbol{\alpha}$

$\beta$

$\gamma$

$\boldsymbol{\delta}$

$\mathbf{l}$

qualifier for interface formed by the intersection of wetting and solid phases entity qualifier

entity qualifier

entity qualifier

entity qualifier

entity qualifier

entity qualifier

\section{Symbols}

- $\quad$ above a superscript refers to a density weighted macroscale average

$=\quad$ above a superscript refers to a uniquely defined macroscale average

, vector tangent to a surface

" vector tangent to a common curve

$\wedge \quad$ identifies a constitutive coefficient

\section{Acknowledgments}

This work was supported by National Science Foundation grant ATM-0941235, Department of Energy grant DESC0002163, and National Institute of Environmental Health Sciences grant P42 ES05948.

\section{References}

1. Al-Raoush RI, Willson C. A pore-scale investigation of a multiphase porous media system. Journal of Contaminant Hydrology 2005;77:67-89. [PubMed: 15722173]

2. Al-Raoush RI, Willson CS. Extraction of physically realistic pore network properties from threedimensional synchrotron X-ray microtomography images of unconsolidated porous media systems. Journal of Hydrology 2005;300(1-4):44-64.

3. Alts T, Hutter K. Continuum description of the dynamics and thermodynamics of phase boundaries between ice and water. Part I: Surface balance laws and their interpretation in terms of threedimensional balance laws averaged over the phase change boundary layer. Journal of NonEquilibrium Thermodynamics 1988;13:221-257.

4. Anderson TB, Jackson R. A fluid mechanical description of fluidized beds. Industrial and Engineering Chemistry Fundamentals 1967;6:527-539.

5. Argentini R, Bakker A, Lowe C. Efficiently using memory in lattice Boltzmann simulations. Future Generation Computer Systems 2004;20(6):973-980.

6. Bachmat Y. Spatial macroscopization of processes in heterogeneous systems. Israel Journal of Technology 1972;10:391-403.

7. Bijeljic B, Blunt MJ. Pore-scale modeling of transverse dispersion in porous media. Water Resources Research 2007;43(12)

8. Bondino I, McDougall SR, Hamon G. Pore network modeling of heavy-oil depressurization: A parametric study of factors affecting critical gas saturation and three-phase relative permeabilities. Spe Journal 2005;10(2):196-205.

9. Bryant SL, King PR, Mellor DW. Network model evaluation of permeability and spatial correlation in a real random sphere packing. Transport in Porous Media 1993;11(1):53-70.

10. Chen C, Packman AI, Gaillard JF. Pore-scale analysis of permeability reduction resulting from colloid deposition. Geophysical Research Letters 2008;35(7) doi 10.1029/2007GL033077. 
11. Cintra JS Jr, Tucker CL III. Orthotropic closure approximations for flow-induced fiber orientation. The Society of Rheology 1995 November/December;39(6):1095-1122.

12. Coles ME, Hazlett RD, Muegge EL, Jones KW, Andrews B, Dowd B, Siddons P, Peskin A, Spanne P, Soll W. Developments in synchrotron X-ray microtomography with applications to flow in porous media. SPE Reservoir Evaluation and Engineering 1998;1(4):288-296.

13. Coles ME, Hazlett RD, Spanne P, Soll WE, Muegge EL, Jones KW. Pore level imaging of fluid transport using synchrotron X-ray microtomography. Journal of Petroleum Science and Engineering 1998;19(1-2):55-63.

14. Crouse B, Rank E, Krafczyk M, Tölke J. A LB-based approach for adaptive flow simulations. International Journal of Modern Physics B 2003;17(1-2):109-112.

15. d'Humiéres D, Ginzburg I, Krafczyk M, Lallemand P, Luo LS. Multiple-relaxation-time lattice Boltzmann models in three dimensions. Philosophical Transactions of the Royal Society of London Series A-Mathematical Physical and Engineering Sciences 2002;360:437-451.

16. Dillard LA, Essaid HI, Blunt MJ. A functional relation for field-scale nonaqueous phase liquid dissolution developed using a pore network model. Journal of Contaminant Hydrology 2001;48:89-119. [PubMed: 11291483]

17. Ding H, Spelt PDM, Shu C. Diffuse interface model for incompressible two-phase flows with large density ratios. Journal of Computational Physics 2007;226(2):2078-2095.

18. Gibbs SJ, Hall LD. What roles are there for magnetic resonance imaging in process tomography? Measurement Science \& Technology 1996;7(5):827-837.

19. Ginzburg I. Equilibrium-type and link-type lattice Boltzmann models for generic advection and anisotropic-dispersion equation. Advances in Water Resources 2005;28(11):1171-1195.

20. Gladkikh M, Bryant S. Prediction of interfacial areas during imbibition in simple porous media. Advances in Water Resources 2003;26(6):609-622.

21. Gray WG. Thermodynamics and constitutive theory for multiphase porous-media flow considering internal geometric constraints. Advances in Water Resources 1999;22(5):521-547.

22. Gray WG, Hassanizadeh SM. Averaging theorems and averaged equations for transport of interface properties in multiphase systems. International Journal of Multiphase Flow 1989;15(1): 81-95.

23. Gray WG, Hassanizadeh SM. Macroscale continuum mechanics for multiphase porous-media flow including phases, interfaces, common lines and common points. Advances in Water Resources 1998;21(4):261-281.

24. Gray WG, Lee PCY. On the theorems for local volume averaging of multiphase systems. International Journal of Multiphase Flow 1977;3:333-340.

25. Gray WG, Miller CT. Thermodynamically constrained averaging theory approach for modeling flow and transport phenomena in porous medium systems: 1. Motivation and overview. Advances in Water Resources 2005;28(2):161-180.

26. Gray WG, Miller CT. Thermodynamically constrained averaging theory approach for modeling flow and transport phenomena in porous medium systems: 3 . Single-fluid-phase flow. Advances in Water Resources 2006;29(11):1745-1765.

27. Gray WG, Miller CT. Thermodynamically constrained averaging theory approach for modeling flow and transport phenomena in porous medium systems: 5. Single-fluid-phase transport. Advances in Water Resources 2009;32(5):681-711.

28. Gray WG, Miller CT. Thermodynamically constrained averaging theory approach for modeling flow and transport phenomena in porous medium systems: 7. Single-phase megascale flow models. Advances in Water Resources 2009;32(8):1121-1142. [PubMed: 20436941]

29. Gray, WG.; Leijnse, A.; Kolar, RL.; Blain, CA. Mathematical Tools for Changing Spatial Scales in the Analysis of Physical Systems. Boca Raton: CRC Press; 1993.

30. Gray WG, Tompson AFB, Soll WE. Closure conditions for two-fluid flow in porous media. Transport in Porous Media 2002;47(1):29-65.

31. Gupta M, Wang KK. Fiber orientation and mechanical properties of short-fiber-reinforced injection-molded composites: Simulated and experimental results. Polymer Composites 1993 October;14(5):367-382. 
32. Hammer EA, Johansen GA. Process tomography in the oil industry - state of the art and future possibilities. Measurement \& Control 1997;30(7):212-216.

33. He XY, Chen SY, Zhang RY. A lattice Boltzmann scheme for incompressible multiphase flow and its application in simulation of Rayleigh-Taylor instability. Journal of Computational Physics 1999;152(2):642-663.

34. Helland JO, Skjaeveland SM. Three-phase mixed-wet capillary pressure curves from a bundle of triangular tubes model. Journal of Petroleum Science and Engineering 2006;52(1-4):100-130.

35. Hilpert M, McBride JF, Miller CT. Investigation of the residual-funicular nonwetting-phasesaturation relation. Advances in Water Resources 2000;24(2):157-177.

36. Hou J. Network modeling of residual oil displacement after polymer flooding. Journal of Petroleum Science and Engineering 2007;59:321-332.

37. Ishii M, Kim S. Development of one-group and two-group interfacial area transport equation. Nuclear Science and Engineering 2004;146:1-17.

38. Ishii M, Kim S, Kelly J. Development of interfacial area transport equation. Nuclear Engineering and Technology 2005;37(6):525-536.

39. Jackson ABS, Miller CT, Gray WG. Thermodynamically constrained averaging theory approach for modeling flow and transport phenomena in porous medium systems: 6 . Two-fluid-phase flow. Advances in Water Resources 2009;32(6):779-795.

40. Johns ML, Gladden LF. Magnetic resonance imaging study of the dissolution kinetics of octanol in porous media. Journal of Colloid and Interface Science 1999;210(2):261-270. [PubMed: 9929413]

41. Kalaydjian F. A macroscopic description of multiphase flow in porous media involving spacetime evolution of fluid/fluid interface. Transport in Porous Media 1987;2:537-552.

42. Kikkinides ES, Yiotis AG, Kainourgiakis ME, Stubos AK. Thermodynamic consistency of liquidgas lattice Boltzmann methods: Interfacial property issues. Physical Review E 2008;78(3, Part 2)

43. Kim, S. PhD thesis. West Lafayette, Indiana: Purdue University; 1999. Interfacial Area Transport Equation and Measurement of Local Interfacial Characteristics.

44. Kulkarni R, Watson AT, Nordtvedt JE. Estimation of porous media flow functions using NMR imaging data. Magnetic Resonance Imaging 1998;16(5-6):707-709. [PubMed: 9803946]

45. Li H, Pan C, Miller CT. Pore-scale investigation of viscous coupling effects for two-phase flow in porous media. Physical Review E 2005;72(2):1-14. 026705.

46. Lowry MI, Miller CT. Pore-scale modeling of nonwetting-phase residual in porous media. Water Resources Research 1995;31(3):455-473.

47. Mahmud WM, Nguyen VH. Effects of snap-off in imbibition in porous media with different spatial correlations. Transport in Porous Media 2006;64(3):279-300.

48. Marle C. Ècoulements monophasiques en milieu poreux. Revue de L'Institut Français du Péetrole 1967 October;22(10):1471-1509.

49. Marle CM. On macroscopic equations governing multiphase flow with diffusion and chemical reactions in porous media. International Journal of Engineering Science 1982;20(5):643-662.

50. Mattila K, Hyvaeluoma J, Timonen J, Rossi T. Comparison of implementations of the latticeBoltzmann method. Computers \& Mathematics with Applications 2008;55(7):1514-1524.

51. Mavriplis DJ. Multigrid solution of the steady-state lattice Boltzmann equation. Computers \& Fluids 2006;35(8-9):793-804.

52. Miller, CA.; Neogi, P. Interfacial Phenomena. New York: Marcel Dekker; 1985.

53. Miller CT, Gray WG. Thermodynamically constrained averaging theory approach for modeling flow and transport phenomena in porous medium systems: 2. Foundation. Advances in Water Resources 2005;28(2):181-202.

54. Miller CT, Gray WG. Thermodynamically constrained averaging theory approach for modeling flow and transport phenomena in porous medium systems: 4. Species transport fundamentals. Advances in Water Resources 2008;31(3):577-597. [PubMed: 19255613]

55. Mirzaei M, Das DB. Dynamic effects in capillary pressure-saturations relationships for two-phase flow in 3D porous media: Implications of micro-heterogeneities. Chemical Engineering Science 2007;62(7):1927-1947. 
56. Moeckel GP. Thermodynamics of an interface. Archive for Rational Mechanics and Analysis 1975;57:255-280.

57. Niessner J, Hassanizadeh SM. A model for two-phase flow in porous media including fluid-fluid interfacial area. Water Resources Research 2008;44 (W08439):doi:10.1029/2007WR006721.

58. Niessner J, Hassanizadeh SM. Non-equilibrium interphase heat and mass transfer during two-phase flow in porous media - Theoretical considerations and modeling. Advances in Water Resources 2009;32:1756-1766.

59. Niessner J, Hassanizadeh SM. Modeling kinetic interphase mass transfer for two-phase flow in porous media including fluid-fluid interfacial area. Transport in Porous Media 2009;80:329-344.

60. Niessner, J.; Hassanizadeh, SM. Two-phase flow and transport in porous media including fluidfluid interfacial area. In: Martin, S.; Williams, JR., editors. Multiphase Flow Research. Nova Science Publishers; 2009. p. 709-729.

61. Nordhaug HF, Celia M, Dahle HK. A pore network model for calculation of interfacial velocities. Advances in Water Resources 2003;26(10):1061-1074.

62. Pan C, Hilpert M, Miller CT. Pore-scale modeling of saturated permeabilities in random sphere packings. Physical Review E 2001;64(6):9.

63. Pan C, Hilpert M, Miller CT. Lattice-Boltzmann simulation of two-phase flow in porous media. Water Resources Research 2004;40(1)

64. Pan C, Luo L-S, Miller CT. An evaluation of lattice Boltzmann schemes for porous medium flow simulation. Computers \& Fluids 2006;35(8-9):898-909.

65. Piri M, Blunt MJ. Three-dimensional mixed-wet random pore-scale network modeling of two- and three-phase flow in porous media. I. Model description. Physical Review E (Statistical, Nonlinear, and Soft Matter Physics) 2005;71(2) 26301-1-30.

66. Premnath KN, Abraham J. Three-dimensional multi-relaxation time (MRT) lattice-Boltzmann models for multiphase flow. Journal of Computational Physics 2007;224(2):539-559.

67. Qin R. Mesoscopic interparticle potentials in the lattice Boltzmann equation for multiphase fluids. Physical Review E 2006;73(6, Part 2)

68. Reeves PC, Celia MA. A functional relationship between capillary pressure, saturation, and interfacial area as revealed by a pore-scale network model. Water Resources Research 1996;32(8): 2345-2358.

69. Sbragaglia M, Benzi R, Biferale L, Succi S, Sugiyama K, Toschi F. Generalized lattice Boltzmann method with multirange pseudopotential. Physical Review E 2007;75(2)

70. Schaap MG, Porter ML, Christensen BSB, Wildenschild D. Comparison of pressure-saturation characteristics derived from computed tomography and lattice Boltzmann simulations. Water Resources Research 2007;43

71. Shan X, Chen H. Lattice Boltzmann model for simulating flows with multiple phases and components. Physical Review E 1993;47:1815-1819.

72. Sheppard AP, Arns JY, Knackstedt MA, Pinczewski WV. Volume conservation of the intermediate phase in three-phase pore-network models. Transport in Porous Media 2005;59(2):155-173.

73. Singh R, Chao JH, Popescu M, Tai CF, Mei RW, Shyy W. Multiphase/multidomain computations using continuum and lattice-Boltzmann methods. Journal of Aerospace Engineering 2006;19(4): 288-295.

74. Slattery JC. Single-phase flow through porous media. American Institute of Chemical Engineers Journal 1969;15(6):866-872.

75. Sochi T, Blunt MJ. Pore-scale network modeling of Ellis and Herschel-Bulkley fluids. Journal of Petroleum Science and Engineering 2008;60(2):105-124.

76. Spanne P, Thovert JF, Jacquin CJ, Lindquist WB, Jones KW, Adler PM. Synchrotron computed microtomography of porous-media - Topology and transports. Physical Review Letters 1994;73(14):2001-2004. [PubMed: 10056943]

77. Sukop MC, Or D. Invasion percolation of single component, multi-phase fluids with lattice Boltzmann models. Physical B-Condensed Matter 2003;338(1-4):298-303. 
78. Sukop MC, Huang H, Lin CL, Deo MD, Oh K, Miller JD. Distribution of multiphase fluids in porous media: Comparison between lattice Boltzmann modeling and micro-x-ray tomography. Physical Review E 2008;77(2)

79. Thauvin F, Mohanty KK. Network modeling of non-Darcy flow through porous media. Transport in Porous Media 1998;31(1):19-37.

80. Thoemmes G, Becker J, Junk M, Vaikuntam AK, Kehrwald D, Klar A, Steiner K, Wiegmann A. A lattice Boltzmann method for immiscible multiphase flow simulations using the level set method. Journal of Computational Physics 2009;228(4):1139-1156.

81. Tölke J, Krafczyk X, Schulz M, Rank E. Lattice Boltzmann simulations of binary fluid flow through porous media. Philosophical Transactions of the Royal Society of London Series AMathematical Physical and Engineering Sciences 2002;360(1792):535-545.

82. Tölke J, Freudiger S, Krafczyk M. An adaptive scheme using hierarchical grids for lattice Boltzmann multi-phase flow simulations. Computers \& Fluids 2006;35(8-9):820-830.

83. Toye D, Marchot P, Crine M, Lhomme G. Modelling of multiphase flow in packed beds by computer-assisted X-ray tomography. Measurement Science \& Technology 1996;7(3):436-443.

84. Wang JY, Zhang XX, Bengough AG, Crawford JW. Domain-decomposition method for parallel lattice Boltzmann simulation of incompressible flow in porous media. Physical Review E 2005;72(1)

85. Whitaker S. Advances in theory of fluid motion in porous media. Industrial and Engineering Chemistry 1969;61(12):14-28.

86. Wildenschild D, Culligan KA, Christensen BSB. Application of X-ray microtomography to environmental fluid flow problems. Developments in X-Ray Tomography IV 2004;5535:432-441.

87. Wildenschild D, Hopmans JW, Rivers ML, Kent AJR. Quantitative analysis of flow processes in a sand using synchrotron-based X-ray microtomography. Vadose Zone Journal 2005;4(1):112-126.

88. Williams RA, Xie CG. Tomographic techniques for characterizing particulate processes. Particle \& Particle Systems Characterization 1993;10(5):252-261.

89. Willson CS, Stacey RW, Ham K, Thompson KE. Investigating the correlation between residual nonwetting phase liquids and pore-scale geometry and topology using synchrotron X-ray tomography. Developments in X-Ray Tomography IV 2004;5535:101-111.

90. Zhao WS, Ioannidis MA. Effect of NAPL film stability on the dissolution of residual wetting NAPL in porous media: A pore-scale modeling study. Advances in Water Resources 2007;30:171-181. 